\title{
Adverse Cardiac Remodelling after Acute Myocardial Infarction: Old and New Biomarkers
}

\author{
Alexander E. Berezin $(\mathbb{D})^{1}$ and Alexander A. Berezin $\left.{ }^{2}\right)^{2}$ \\ ${ }^{1}$ Internal Medicine Department, State Medical University, Ministry of Health of Ukraine, Zaporozhye 69035, Ukraine \\ ${ }^{2}$ Internal Medicine Department, Medical Academy of Post-Graduate Education, Ministry of Health of Ukraine, \\ Zaporozhye 69096, Ukraine
}

Correspondence should be addressed to Alexander E. Berezin; aeberezin@gmail.com

Received 15 May 2019; Revised 6 January 2020; Accepted 22 May 2020; Published 12 June 2020

Academic Editor: Roberta Rizzo

Copyright (c) 2020 Alexander E. Berezin and Alexander A. Berezin. This is an open access article distributed under the Creative Commons Attribution License, which permits unrestricted use, distribution, and reproduction in any medium, provided the original work is properly cited.

\begin{abstract}
The prevalence of heart failure (HF) due to cardiac remodelling after acute myocardial infarction (AMI) does not decrease regardless of implementation of new technologies supporting opening culprit coronary artery and solving of ischemia-relating stenosis with primary percutaneous coronary intervention (PCI). Numerous studies have examined the diagnostic and prognostic potencies of circulating cardiac biomarkers in acute coronary syndrome/AMI and heart failure after AMI, and even fewer have depicted the utility of biomarkers in AMI patients undergoing primary PCI. Although complete revascularization at early period of acute coronary syndrome/AMI is an established factor for improved short-term and long-term prognosis and lowered risk of cardiovascular (CV) complications, late adverse cardiac remodelling may be a major risk factor for one-year mortality and postponded heart failure manifestation after PCI with subsequent blood flow resolving in culprit coronary artery. The aim of the review was to focus an attention on circulating biomarker as a promising tool to stratify AMI patients at high risk of poor cardiac recovery and developing HF after successful PCI. The main consideration affects biomarkers of inflammation, biomechanical myocardial stress, cardiac injury and necrosis, fibrosis, endothelial dysfunction, and vascular reparation. Clinical utilities and predictive modalities of natriuretic peptides, cardiac troponins, galectin 3, soluble suppressor tumorogenicity-2, high-sensitive C-reactive protein, growth differential factor-15, midregional proadrenomedullin, noncoding RNAs, and other biomarkers for adverse cardiac remodelling are discussed in the review.
\end{abstract}

\section{Introduction}

Heart failure (HF) is a global health problem with serious economic burden that has been considered as the dominant cause of cardiovascular (CV) morbidity and mortality in the developed and developing countries $[1,2]$. HF affects around 26 million people worldwide (including 5.7 million and 3.4 million people in the US and in the EU, respectively), and the estimated expenditures for HF care were around $\$ 31$ billion [1]. It is expected that by 2030 more than 40 million people will have this condition, and the HF diagnosis and therapy will increase twice and even more [3]. The clinical outcomes remain poor with a five-year survival rate of approximately $50 \%$ regardless of phenotype of HF that completely correspond to the expected survival rate in non- metastatic cancer [3, 4]. Despite sufficient improvements in diagnosis, prevention and treatment of HF new incidences of $\mathrm{HF}$ with reduced ejection fraction (HFrEF) and midrange ejection fraction (HFmrEF) in contrast to HF with preserved ejection fraction (HFpEF) continue to occur as a need for heart transplantation and mechanical support device use [4]. Additionally, increased prevalence of HFpEF represents the most frequent cause of $\mathrm{CV}$ and sudden death, primary hospitalization, and readmission to the hospital due to acute decompensation of HF [5].

The most common primary causes of $\mathrm{HFrEF} / \mathrm{HFmrEF}$ remain acute ST-segment elevation myocardial infarction (STEMI) and hypertension, while incidences of HFpEF were rather associated with hypertension, acute non-ST-segment elevation myocardial infarction (non-STEMI), and 
alternative causes (atrial fibrillation, cardiomyopathy, myocarditis, valvular heart disease, and diabetes mellitus) compared with STEMI [6-8]. Contemporary strategy for the prevention of HF after acute STEMI is based on early complete cardiac revascularization and prevention of negative impact of comorbidities, such as diabetes mellitus, abdominal obesity, hypertension, thyroid dysfunction, kidney failure, and conventional CV risk factors (smoking, dyslipidaemia, insulin resistance, and hyperuricemia) [9, 10]. In fact, complete recovering of blood flow through culprit coronary artery and other ischemia-related stenosis with primary percutaneous coronary intervention (PCI) is not warranted for full prevention of late adverse cardiac remodelling $[11,12]$. Although improvement of prognosis, increase in quality of life, and delay in progression or reversal of ischemia-induced cardiac remodelling and chronic $\mathrm{HF}$ remain prime targets for the treatment of AMI $[13,14]$, there are no clear approaches for risk stratification in AMI patients after successful PCI [15]. For instance, hyperemic microcirculatory resistance and no-reflow phenomenon were found as strong predictors for the extent of infarct size and early cardiac remodelling [16]. Additionally, optic coherent tomography or intravascular ultrasound performed over 3 months after initial major cardiac event frequently allows identifying several factors contributing advance in late cardiac remodelling, such as silent restenosis, progression of old stenotic lesions, late stent thrombosis, and several postPCI technical problems with incomplete stent branches' expansion, stent malposition, and underpressed culprit plaque [17, 18]. Except for early revascularization, cardiac remodelling could be prevented by pharmacotherapy including complex neurohormonal blockade and device-based therapies, which are addressed in the improvement of ventricular dyssynchrony and prevention from fatal arrhythmias [19]. In this context, new diagnostic and predictive options are needed to prevent cardiac remodelling and HF. The aim of the review was focused on the circulating biomarker as a tool to stratify postmyocardial infarction patients at high risk of poor cardiac recovery after reperfusion with primary PCI and developing HF.

\section{Adverse Cardiac Remodelling after Acute Myocardial Infarction: Definitions and Contributing Factors}

2.1. Definition of Adverse Cardiac Remodelling. Adverse cardiac remodelling after AMI is defined as complex interactions between cellular and extracellular components of myocardium, which are neurohumoral and epigenetic regulations, leading to changes in the cardiac architectonics and geometry frequently affecting both ventricles and atrials, worsening diastolic filling and systolic function and associated with developing heart failure [17]. Additionally, there is a large number of definitions of cardiac remodelling after STEMI, which are based on multiple imaging modalities, such as presentation of akinesia area, left ventricle enlargement, reduced LVEF, and early diastolic dysfunction (including longitudinal strain increase, twist of LV apex, and tethering effect). In fact, an impact of passive mechanical constraint of surrounding myocardium on infarct zone mediates infarct expansion and decline in both regional and global systolic function [20].

Other criteria of cardiac remodelling affect shaping stunned and hibernated myocardium after incomplete revascularization or delay of PCI performing with inadequate perfusion recovery [21]. However, non-STEMI is also associated with cardiac remodelling, rather mild-to-moderate than severe, and frequently nondistinguished from STEMIinduced cardiac disorders in prognostic aspects, but the canonic model of pathogenesis of adverse cardiac remodelling was based on STEMI impact on cardiac architectonic. There is a sustainable option that STEMI-induced cardiac remodelling frequently relates to $\mathrm{HFrEF} / \mathrm{HFmrEF}$, but nonSTEMI-induced cardiac remodelling is rather associated with developing HFpEF than HFrEF.

2.2. Contributing Factors of Adverse Cardiac Remodelling. In fact, there are at least two different variants of adverse cardiac remodelling after acute $\mathrm{MI}$, which distinguished each other in pathogenesis, so called the early (at 2-3 weeks after initial event) and late (at 3-6 months after AMI) remodelling (see Figure 1).

Contemporary point of view is based on an idea that early complete primary revascularization of culprit artery and ischemia-induced stenosis/occlusions in other coronary arteries at first hours of STEMI is independent and the most powerful factor preventing early LV cavity dilation, declining LV pump function and the developing of HF. It has been postulated that preserved systolic function and LV dimensions at early stage of various revascularization procedures can accompany with myocardial biomechanical and energetics stress, mitochondrial dysfunction, and oxidative stress that lead to potent fatal arrhythmias even prior to diastolic dysfunction developing [22]. Over the next three months after restoring TIMI III blood flow through culprit artery with PCI, the primary causes inducing adverse cardiac remodelling can be different from the aforementioned. Indeed, other factors that may contribute to cardiac remodelling after successful primary PCI are arterial healing, vessel remodelling, stent restenosis, thrombosis, and incomplete expansion of stent branches (known as malposition), and stent fracture, which require ischemia-driven target vessel revascularization further [23]. Performing of optical coherence tomography (OCT) in STEMI patients presenting with late and very late stent thrombosis has yielded that stent malposition was determined in 55\% cases, quarter of which had been found evidence of positive vessel remodelling [24]. Additionally, neoatherosclerosis and uncovered stent struts were reported as the primary cause of late thrombosis in 35\% cases and 10\% cases, respectively [24]. Although coronary stent fracture is an underrecognized event, it has been reported frequently in the drug-eluting stent era [25]. However, investigators have shown that technical problems with first-generation eluting stent implantations in STEMI patients were associated with higher inhospital mortality and posthospital target vessel failure or cardiac death [24]. 


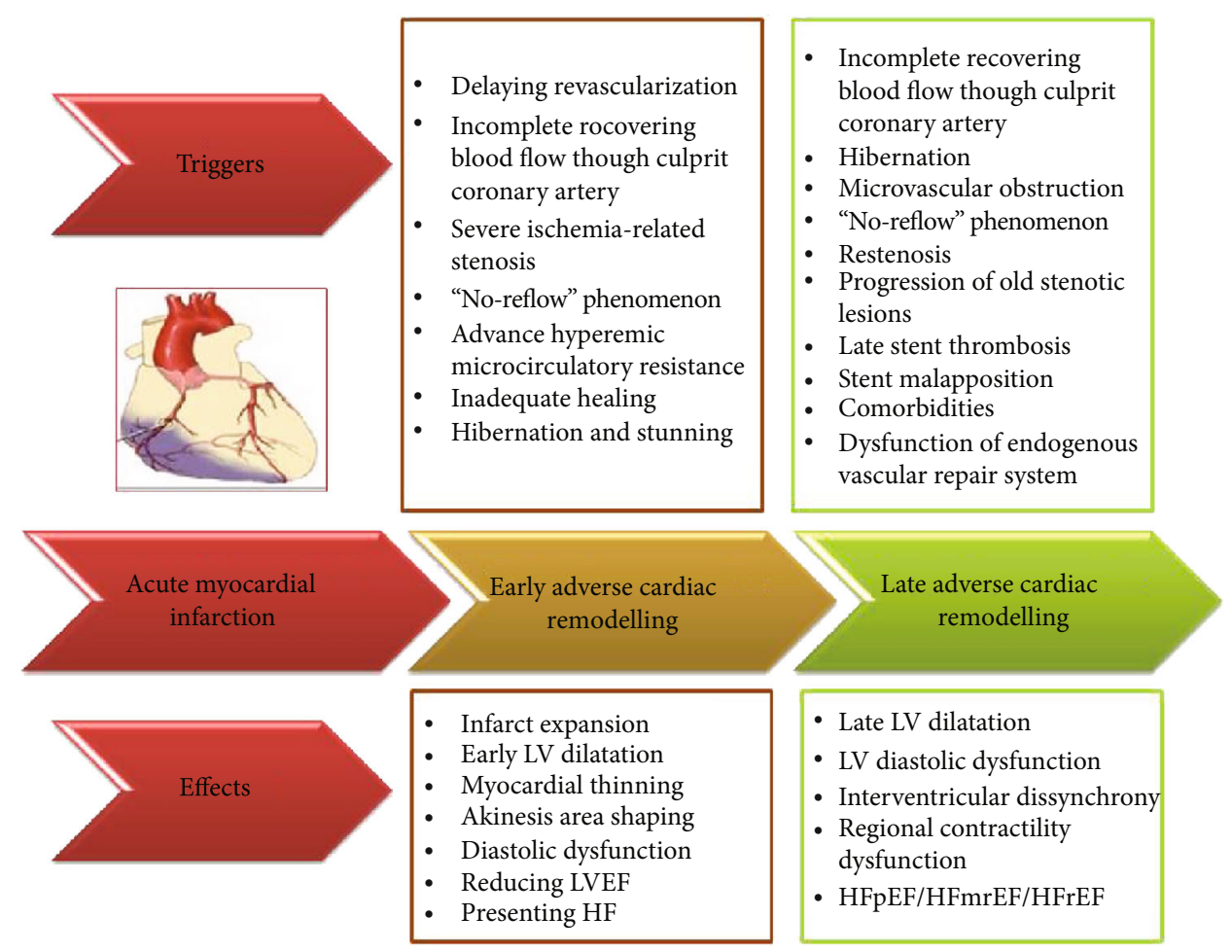

FIGURE 1: Adverse cardiac remodelling after AMI: the role of different triggers in development of cardiac architectonic disorders and heart failure. LV: left ventricular; HF: heart failure; HFpEF: HF with preserved ejection fraction; HFmrEF: HF with midrange ejection fraction; HFrEF: HF with reduced ejection fraction.

Endothelial shear stress, neointima formation, and late thrombosis can appear beyond inadequate PCI and stent positioning and are result of accelerating atherosclerosis and inadequate drug support, i.e., nonoptimal care with statins, refusal from dual antiplatelet therapy, effective anticoagulation if needed, and adenosine intracoronary for prevention no-reflow/slow-flow phenomena. Even a novel device (known as bioabsorbable cardiac matrix) was not able to attenuate adverse cardiac remodelling after AMI [26], while there were strong positive expectations regarding these devices [27]. Despite implantation of second-generation everolimus-eluting stent in STEMI appears to be better to first-generation eluting stents, there is evidence that even a small degree of chronic intrastent conditions may significantly influence on healing persistence [28]. Frequencies of uncovered and malapposed struts as well as percentage of stents fully covered with neointima were $1.2 \%, 0.4 \%$, and $60.9 \%$, respectively, for over a one-year period after PCI with second-generation everolimus-eluting stent implantation [28]. In fact, they were not associated with the incidence of clinical events and intrastent thrombus.

The next factor contributing to early and late cardiac remodelling is the "no-reflow" phenomenon. Indeed, the "no-reflow" phenomenon can be considered as a component of early cardiac remodelling after STEMI that relates to microvascular obstruction and dysfunction causing severe disturbance in regional perfusion [29]. In fact, the "noreflow" phenomenon is a result in poor healing of the culprit artery and adverse cardiac remodelling, increasing the risk for major adverse cardiac events, such as recurrent MI, newly diagnosed HF, and sudden death, but the "slow-flow" phenomenon appears to be a serious factor contributing to both types of adverse cardiac remodelling [30, 31].

Additional factor that is involved onto a development of late adverse remodelling is epigenetically mediating disturbance of endogenous vascular repair system $[32,33]$. It has been found that altered vascular repair has maintained vasoconstriction and vascular dysfunction that accelerated atherosclerosis and supported hibernation in the grey zone around myocardial infarction. Overall, the development of adverse cardiac remodelling after AMI regardless of initial cause (even in asymptomatic patients) was consistently associated with poor clinical outcomes, and it could be predicted and completely resolved [34, 35].

The factors preventing late adverse cardiac remodelling after successful reperfusion with primary PCI in STEMI patients are indicated in Figure 2. Recognition of the heterogeneous pathophysiology of adverse cardiac remodelling after AMI can create a powerful risk stratification score based on biomarkers reflecting various stages of pathogenesis of the condition [36].

\section{Pathogenetic Mechanisms of Adverse Cardiac Remodelling after Acute Myocardial Infarction}

Advances in our understanding of the molecular mechanisms of regulation toward late adverse cardiac remodelling were associated with the breakthrough in the recognition of 
Prevention of late adverse cardiac remodelling

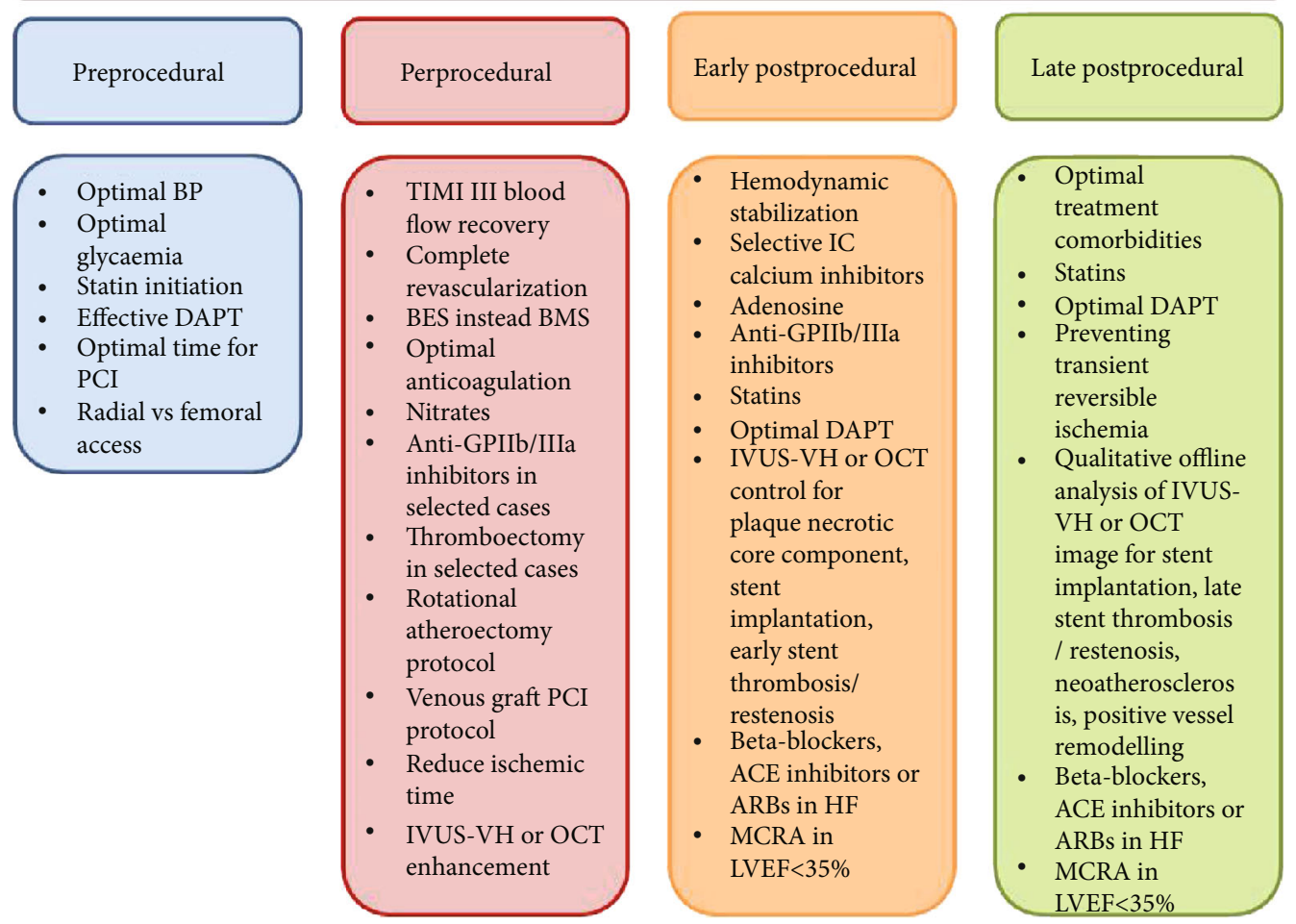

FIgURE 2: The factors preventing late adverse cardiac remodelling in AMI patients after successful reperfusion with PCI. IVUS-VH: intravascular ultrasound virtual-histology; BMS: bare metal stent; BES: biolimus eluting stent; OCT: optical coherence tomography; DAPT: dual antiplatelet therapy; ACE: angiotensin-converting enzyme; ARBs: angiotensin-II receptor antagonists; MCRA: mineralocorticoid receptor antagonists; IC: intracoronary.

interplaying between various processes translating ischemia/reperfusion injury on myocardium, such as disrupting nitric oxide (NO) and vascular endothelial growth factor (VEGF) signalling systems, p38 MAPK pathway and redox dysregulation, cytokine release, and activation of apoptotic and necrotic death pathways with subsequent stimulation of oxidative stress, mitochondrial dysfunction, altered myocardial cell metabolism, excessive fibrosis, and cardiac cell remodelling [37]. Therefore, preserved microvascular inflammation, small vessel obstruction, endothelial dysfunction, and atherosclerotic lesions mediate a remote effect on advance LV remodelling [38]. Additionally, there are new explanations regarding individual susceptibility to ischemia/reperfusion injury including early and remote ischemic preconditioning [39]. Figure 3 yields main pathogenetic mechanisms that are involved in the pathogenesis of late adverse cardiac remodelling.

In fact, restoration of adequate blood perfusion after a critical period of ischemia and prevention of reperfusion damage appear to be not the only protector over cardiac damage. Early irreversible cardiac myocyte injury leading to necrosis in the ischemic myocardium and expanding infarction zone are an attribute of susceptibility of cardiac cells to impaired metabolism, loss of structural integrity and selective permeability of the cell membranes, altered ultrastructure of cell organoids, such as sarcolemma disruption, deterioration of nucleus, ribosomes, mitochondria, and sar- coplasmic reticulum, the presentation of mitochondrial amorphous densities, and chromatin fragmentation [40]. During this early stage of AMI development, the mitochondrial dysfunction plays a pivotal role in cardiac myocyte apoptosis in the ischemic/reperfused heart, cardiac necrosis, and ischemia-induced preconditioning phenomenon [41, 42].

Numerous studies have shown that proapoptotic stimuli through involving cytokines, which belong to the B-cell lymphoma 2 (Bcl-2) super family, mediate the permeability of the mitochondrial membranes and stimulate the release of a wide spectrum of the active apoptogenic molecules (cytochrome c, Bax) into the cytoplasm. They cause the apoptotic response, peroxidation of membrane, and disruption of mitochondrial chromatin materials, including small interfering ribonucleic acid (RNA) and mitochondrial deoxyribonucleic acid (DNA) [43, 44]. Cytochrome $\mathrm{C}$ is able to bind to the adaptor protein apoptotic protease activating factor 1 (Apaf-1) and act as a trigger of its oligomerization that activates caspase cascade through initiating procaspase- 9 recruitment. Caspases including caspase6 and caspase- 9 cleave cellular proteins and DNAs/RNAs emerging apoptosis [45]. This process is under the close epigenetic regulation of long noncoding RNAs (LncRNA) and microRNAs (miRNA-29b-1-5p, miRNA-195), which negatively regulate $\mathrm{Bcl} 2 \mathrm{l} 2$ gene expression and participate in cardiac myocyte apoptosis, oxidative stress through inducing hydrogen peroxide $\left(\mathrm{H}_{2} \mathrm{O}_{2}\right)$, and inflammation 


\begin{tabular}{|c|c|c|}
\hline $\begin{array}{l}\text { Acute MI/ Acute } \\
\text { coronary syndrome }\end{array}$ & $\begin{array}{l}\text { Early period after } \\
\text { successful PCI }\end{array}$ & $\begin{array}{l}\text { Remote period } \\
\text { after PCI }\end{array}$ \\
\hline $\begin{array}{l}\text { First hours before } \\
\text { restoration of coronary } \\
\text { circulation }\end{array}$ & Within 3 months after PCI & Over 3 months after PCI \\
\hline Myocardial necrosis & Distal embolization & Remote conditioning \\
\hline Ischemia/reperfusion damage & $\begin{array}{l}\text { Stunning/hubernation } \\
\text { no-reflow/slow-flow }\end{array}$ & Atherosclerosis progression \\
\hline Expanding necrosis & Transient ischemia/reperfusion & Hibernating myocardium \\
\hline Preconditioning & Postconditioning & $\begin{array}{c}\text { Remote ischemia/reperfusion } \\
\text { episodes }\end{array}$ \\
\hline $\begin{array}{c}\text { Severity of coronary } \\
\text { atherosclerosis }\end{array}$ & Post-PCI technical problems & Inflammation and fibrosis \\
\hline Comorbidities & Atherosclerosis progression & ECM and cell remodelling \\
\hline Preexisting HF & Inflammation and fibrosis & $\begin{array}{c}\text { Impaired cardia and vascular } \\
\text { reparation }\end{array}$ \\
\hline Individual susceptibility & $\begin{array}{l}\text { Impaired cardiac and vascular } \\
\text { reparation }\end{array}$ & Comorbidities \\
\hline
\end{tabular}

FIGURE 3: The main pathogenetic mechanisms underlying the initiation and progression of late adverse cardiac remodelling in AMI patients after successful reperfusion with PCI. HF: heart failure; ECM: extracellular matrix; PCI: percutaneous coronary intervention.

via triggering proinflammatory cytokine release $[44,46]$. Therefore, downregulated miRNA-98 and miRNA-124 may attenuate cell survival through diminished levels of STAT3 and p-STAT3 in response to ischemia and over production of $\mathrm{H}_{2} \mathrm{O}_{2}[47,48]$. During ischemia/reperfusion episodes, oxidative stress, mitochondrial $\mathrm{Ca}^{2+}$ overload, proinflammatory cytokines (interleukin- (IL-) 2, IL-6, tumor necrosis factoralpha, and interferon-gamma) stimulate the activity of the matrix metalloproteinases and suppress release of their tissue inhibitors [49]. MMPs (MMP-2, MMP-6, and MMP-9) directly contribute to global and local myocardial contractile dysfunction and induce cell death [50]. Other matricellular proteins, such as thrombospondin- (TSP-) 1 and TSP-2, as well as bone-related proteins (osteopontin, osteonectin, and osteoprotegerin), were found to regulate cardiac reparation and remodelling via activation of VEGF and transforming growth factor (TGF- $\beta$ ) by binding to the latency-associated propeptide, inhibition of MMP activity, and exertion of potent angiostatic actions of antigen-presenting cells and T-cells [51]. Moreover, they are triggers for accumulation, degradation and remodelling of extracellular matrix (ECM), cleaving big endothelin- 1 and attenuating vasoconstriction, and modification of architectonics of myocardium leading to cardiac remodelling and HF development $[51,52]$.

Interestingly, susceptibility of myocardium to ischemia and reperfusion may relate to various inhered causes, such as mutations in genes encoding for angiotensin II, angiotensin-converting enzyme, osteopontin, osteoprotegerin, CC chemokine receptor 2, the members of the family of multidomain extracellular protease enzymes ADAMTS (A Disintegrin and Metalloproteinase with Thrombospondin motifs), predominantly ADAMTS-2, ADAMTS-4,
ADAMTS-10, and ADAMTS-13, promoter region of endothelial NO synthase, apelin, TGF- $\beta$, VEGF, galectin-3, ficolin-1, S100 calcium-binding protein A9, and mitochondrial aldehyde dehydrogenase 2 (NDUFC2) [53-56]. Finally, susceptibility of cardiac cells to ischemia and reperfusion damage may relate to the capability of endogenous redox systems to protect cell membranes and cellular structures (mitochondria, cytoskeletal proteins, growth factor receptors, and microtubule-associated proteins) from the impaired effect of the deteriorating energetic metabolism and detergenting impact of oxidized lipids and proteins sustaining an effective work of transmembrane ionic pumps [57]. This phenomenon was called ischemic preconditioning, and now it is also recognized as an early (before AMI or during acute phase of AMI) and remote (overreparative period of AMI) phenomenon depending on a period of onset of ischemia-reperfusion episodes. However, previous studies have revealed a reduction of infarct size and peripheral area with hibernating/stunning myocardium with both types of preconditioning due to intracardiac protection that prevents cytosolic and mitochondrial $\mathrm{Ca}^{2+}$ overload, accumulation of reactive oxygen species (ROS), lysosomal/nonlysosomal enzyme releasing, and inflammatory reaction [58-60].

Recurrent episodes of ischemia/reperfusion induce cardioprotective mechanisms in failed heart named postconditioning and remote conditioning $[61,62]$, which are supported by various comorbidities (diabetes mellitus, insulin resistance, obesity, and inflammatory conditions) [63, 64]. The cardiac protective mechanisms may include upregulation of caveolin, resolvin D1/E1, ubiquinone, long pentraxin PTX3, apelin, glucocorticoids, and long noncoding RNAs expression for IL-19, VEGF, eNO synthase, haem 
oxygenase-1 (HO-1), calcitonin gene-related peptide, and peroxisome proliferator-activated receptor gamma, and downregulation of $\beta$-adrenergic signalling, $G$ proteincoupled receptor kinase- 2 , and $\beta$-arrestin 1 and 2 in cardiac myocytes, fibroblasts/myofibroblasts, tissue residence cells, and circulating progenitor cells as well as mononuclears [65-68]. These factors reduce inflammatory infiltrates, stabilize cell membrane, support membrane ionic channels, and suppress the formation of key proinflammatory cytokines, such as tumor necrosis factor-alpha (TNF- $\alpha$ ), IL- $1 \beta$, and IL-6. Additionally, IL-19 suppresses the polarization of proinflammatory subtype M1 macrophages and triggers M2 macrophage polarization in infarct myocardium that leads to inhibition of cardiac remodelling [68].

During AMI and recurrent episodes of micronecrosis in myocardium after PCI due to remote ischemia/reperfusion damage, the important role in the regulation of cardiac remodelling belongs to alarmins, which are released by necrotic myocardium and act as a powerful trigger of inflammatory cytokine synthesis [69]. Damaged and necrotic cardiac myocytes secrete wide-spectrum factors called DAMPs (Damage-Associated Molecular Patterns), such as highmobility group 1B protein (HMGB1), RNA, nucleotides, heat shock proteins (HSP), members of the S100 family, and IL1a, which potentiate the inflammatory response, attenuate oxidative stress, act as direct cytotoxic agents, and induce thrombus formation and circulating blood cell aggregation [70]. Numerous molecules, such as HMGB1, S100 family members, are able to induce apoptosis of circulating endothelial progenitor cells and tissue residence cells via a multiligand receptor for advanced glycation end products(RAGE-) mediated activation of endoplasmic reticulum stress pathway [71]. Therefore, the DAMPs and other chemokines, such as CXC and CC (predominantly CCL2, CCR2, CCR5, and ELR+CXC chemokines), recruit various subpopulations of peripheral blood cells including proinflammatory mononuclears, regulatory T-cells, nature killers, and neutrophils in the infarcted myocardium and endothelium supporting inflammatory response [72].

Inflammation is a crucial element for clearance of cellular and matrix debris, while suppression of proinflammatory signalling is necessary to transform the inflammatory phase to the proliferative phase [73]. Indeed, proinflammatory mediators include uncoupling protein 2, superoxide dismutase- (SOD-) 1 and SOD-2, ROS, through the activation of mTOR, hypoxia-induced factor- (HIF-) 1, Toll-like receptor (TLR)/IL-1, and RAGE-dependent pathways in surviving border-zone fibroblasts, cardiac myocytes, endothelial cells, smooth muscle cells, mononuclears, and several residence and progenitor cells mediating reparative processes [74-76]. It relates to the modification of cardiac fibroblasts into myofibroblasts that are enriched in $\alpha$ smooth muscle actin, accumulation of extracellular matrix, neovascularization, and angiogenesis. However, the proinflammatory cytokines may have a detrimental impact on cardiac remodelling and function directly maintaining repetitive ischemia/reperfusion episodes, suppressing reparation, supporting endothelial dysfunction, coagulation, and thrombosis [77-79].
Over a 3-month period after PCI, the extracellular matrix is continually being remodelled, and tissue fibroblasts, myofibroblasts, and antigen-presenting cells become quiescent and undergo apoptosis, and cell debris is cleared by macrophages [80-82]. The regulation of proliferative response and changing of cellular phases of tissue inflammation are mediated by the renin-angiotensin-aldosterone system (RAAS) and simpatico adrenal system, which also are central players in the endogenous repair system [83, 84]. Additionally, the autonomic nervous system may play a crucial role in the inflammatory and apoptotic remodelling following AMI [85]. Thus, late adverse cardiac remodelling is a sophisticated structural and functional response of failing heart to numerous triggers (inflammation, fibrosis, cell survival signalling, and $\beta$-adrenergic signalling) and damaged factors (ischemia, reperfusion, necrosis, and apoptosis) that appear consequently and mutually activate each other.

\section{Biomarkers of Adverse Cardiac Remodelling}

Although there are well-developed current clinical recommendations for HF provided by the experts of the European Society of Cardiology (2016) [86] and American College of Cardiology/American Heart Association (2017) [87], there is a lack of statements for the use of biomarker strategies for diagnosis, prediction, stratification, and prevention of adverse cardiac remodelling. In fact, cardiac remodelling after AMI regardless of PCI and other approaches for revascularization is strongly associated with the development and progress of HF. In this context, early biomarkers of myocardial injury and necrosis, as well as biomarkers of biomechanical stress, neurohumoral and inflammatory activation, and fibrosis, having predictive and diagnostic evidence for acute and chronic HF, are extrapolated over strategy regarding diagnosis, outcomes, and stratification of adverse cardiac remodelling (Table 1 ).

There is no complete agreement between experts from the European Society of Cardiology and American College of Cardiology/American Heart Association regarding the utility of biomarkers in HF [88]. Natriuretic peptides (NPs) are recommended by both guidelines for acute and chronic HF diagnosis, prediction of HF-relating outcomes, including death, and a risk stratification. In contrast, the European Society of Cardiology (2016) HF clinical recommendation does not consist the supporting evidence regarding other biomarkers for multitask strategy in HF, and HF-guided therapy is not routinely recommended, while the HF biomarker guidance was previously approved by the American College of Cardiology/American Heart Association. Additionally, there was poor discrimination when NPs were used in patients with $\mathrm{HF}$ at hospital discharge, which was inferior to its performance in patients with ambulatory HF regardless of severity cardiac dysfunction and phenotypes.

However, there is a large body of evidence that other biomarkers (growth/differential factor-15, MMP-2, MMP-6, MMP-9, adipocytokines (apelin, chemerin, and visfatin), circulating endothelial and mononuclear progenitor cells, activated and apoptotic endothelial cell-derived microvesicles, miRNAs, and bone-related proteins) reflecting different 
TABLE 1: Clinical relevance of circulating biomarkers for late adverse cardiac remodelling: overlap with HF.

\begin{tabular}{|c|c|c|c|c|c|c|c|}
\hline \multirow{2}{*}{ Biomarkers } & \multicolumn{4}{|c|}{ Heart failure } & \multicolumn{3}{|c|}{ Adverse cardiac remodelling } \\
\hline & Diagnosis & Outcomes & Guided therapy & Risk stratification & Diagnosis & Outcomes & Risk stratification \\
\hline \multicolumn{8}{|c|}{ Currently used or recommended biomarkers } \\
\hline hs-troponin $\mathrm{T} / \mathrm{I}^{\ddagger}$ & - & ++ & - & + & + & + & + \\
\hline $\mathrm{NPs}^{\# *}$ & ++ & +++ & + & ++ & ++ & +++ & ++ \\
\hline MR-proADM & + & +++ & - & ++ & + & +++ & ++ \\
\hline Galectin- $3^{\neq}$ & - & + & - & + & - & ++ & + \\
\hline $\mathrm{sST}^{\neq}$ & - & ++ & + & - & - & +++ & + \\
\hline \multicolumn{8}{|c|}{ Promising biomarkers } \\
\hline Copeptin & + & ++ & - & + & + & ++ & + \\
\hline GDF15 & - & ++ & - & + & - & ++ & ++ \\
\hline hs-CRP & - & + & - & - & - & + & + \\
\hline IL- $1 \beta$ & - & + & - & + & - & + & + \\
\hline IL-6 & - & + & - & + & - & + & + \\
\hline MMP-2 & - & + & - & - & + & + & + \\
\hline MMP-9 & - & + & - & - & + & + & + \\
\hline CTPpC-I & - & + & - & + & - & + & ++ \\
\hline APpC-III & - & + & - & + & - & + & ++ \\
\hline miRNAs & - & + & + & + & - & + & + \\
\hline
\end{tabular}

Mildly disagree; ${ }^{-}$moderately disagree; ${ }^{+}$mildly agree; ${ }^{++}$moderately agree; ${ }^{+++}$strongly agree; ${ }^{*}$ approved by the European Society of Cardiology (2016); ${ }^{7}$ approved by the American College of Cardiology/American Heart Association (2017). hs: high sensitive; HF: heart failure; NPs: natriuretic peptides; sST2: soluble suppression of tumorigenicity-2; MR-proADM: midregional proadrenomedullin; GDF: growth/differential factor; CRP: C-reactive protein; miRNAs: microribonucleic acids; MMP: matrix metalloproteinase; CTPPC-I: carboxytelopeptides of procollagen type I; APpC-III: aminopeptide of procollagen type III.

stages of the pathogenesis of adverse cardiac remodelling after PCI can be considered as promising tools for further strategies to improve prediction of clinical outcomes, attenuate CV risk stratification, and develop personifying strategy for treatment $[89,90]$. For instance, miRNAs are speculated to have crucial roles in the nature evolution of adverse cardiac remodelling after AMI, and identification of key genes associated with damaged heart response could improve prediction models for the patients [91]. Moreover, miRNA profiling and gene cards with information about a signature of mutations involved in the regulation of the transcription factors, which mediate cardiac remodelling, appear to be promising for further precise medicine after PCI [92].

\section{Biomarkers of Cardiac Injury and Necrosis}

Elevated levels of high-specific cardiac troponins T (hs-TnT) and I (hs-TnI) in peripheral blood are served as diagnostic and predictive biomarkers for acute coronary syndromes and AMI [93], as well as an independent prognosticator of CV risk in the general population [94]. Cardiac troponins are structure proteins of actin-myosin complex, which are released from the cells due to necrosis or leakage from cytosol through the permeable cell membrane [95]. High-sensitivity cardiac troponin assay allows diagnosing patients with minor myocardial injury and suggesting a size of infarction [96]. Cell-free pool of cardiac troponins was reported having a tendency to decrease after AMI, while peak concentrations of both hs-TnT and hs-TnI have strongly predicted major cardiovascular events including death, recurrent MI, need of PCI, and subsequent HF hospitalization [96, 97]. Moreover, elevated concentrations of circulating cardiac troponins remain useful independent predictive biomarkers of newly post-AMI HF $[98,99]$. Interestingly, elevated levels of hsTnI were associated with CV death, whereas hs-TnT has more strongly predicted the risk of non-CV death [100]. In fact, cardiac and noncardiac surgeries mediate the elevation of troponins in the peripheral blood postprocedurally. It requires specific approach to assay an impact of transient elevation of these findings on a risk of poor prognosis. Obviously, the combined biomarkers' models are necessary.

After a prolonged period of hopes regarding improvement of diagnostic and risk stratification in STEMI patients with subsequent PCI using the combined biomarker models (cardiac troponins, NPs, copeptin, choline, soluble ST2, GDF-15, high-sensitivity C-reactive protein, galectin-3, and lipoprotein-associated phospholipase A2) [101], it has clearly become what large clinical trials need to evaluate diagnostic and predictive values of various combinations of biomarkers, because the evidence of previous studies in AMI patient treated with PCI appeared to be controversial [102, 103]. Copeptin did not add diagnostic information to peak concentration of high-sensitive troponin $\mathrm{T}$ in STEMI patients with subsequent PCI $[104,105]$. Yet, hs-TnT/hs-TnI and NT-probrain NP (NT-proBNP) were recognized to have similar predictive values for all-cause mortality and first readmission in HFpEF [106, 107], whereas NT-proBNP was superior to cardiac troponins for the prognostication of HFrEF clinical outcomes [108, 109]. It has been noted that the predictive value of hs-TnI for HF-related clinical outcome was strongest in men with $\mathrm{HFpEF} / \mathrm{HFrEF}$ than in women [108]. Other biomarkers, including soluble ST2, 
high-sensitivity C-reactive protein, galectin-3, midregional proadrenomedullin, and GDF-15, in combination with hs-TnI/hs-TnT did not represent superiority in comparison with the isolated use of hs-TnI/hs-TnT in HFpEF, whereas in patients with $\mathrm{HFmrEF} / \mathrm{HFrEF}$, multimarkers' strategy was better in the prognostication of poor prognosis $[110,111]$.

Although previous clinical trials did not find significant interactions between stable HFpEF and HFrEF when considering the prognostic value of the NT-proBNP, cystatinC, hs-TnT, and soluble ST2 [112-114], it can be otherwise for HF that is associated with adverse cardiac remodelling after AMI with subsequent PCI. Thus, clinical prediction models for HF-related outcomes based on various biomarkers of biomechanical stress (NT-proBNP, copeptin, midregional proadrenomedullin (MR-proADM), and growth/differential factor- (GDF-) 15), inflammation (high-sensitivity C-reactive protein), and fibrosis (galectin-3, soluble ST2) were only improved marginally by the addition of hs-TnT/hs-TnI. Moreover, hs-TnT or hs-TnI added to NT-proBNP and sST2 appears to be emerging biomarkers in the prediction of adverse outcome of HF after AMI in a short-term period [115], but whether this combination is most suitable for remote prognostication in patients with known late adverse cardiac remodelling and different phenotypes of ischemiainduced HF is not fully clear.

\section{Inflammatory Biomarkers}

6.1. Interleukins. IL- $1 \beta$, IL-6, and angiopoietin-like protein 2 (Angptl 2) are inflammatory cytokines that influence deleterious effects on myocardium structure and function unleashing to cardiac remodelling [116]. There is strong evidence clarifying that the myocardial expression levels of IL- $1 \beta$, IL6, and Angptl 2 were significantly higher in the AMI patients than in the healthy volunteers [117]. Moreover, the levels of Angptl 2 and IL-6 rather correlated with the severity of coronary atherosclerosis than the size of the infarct area and HF presence. In contrast, IL- $1 \beta$ levels were associated with prior HF admissions, functional cardiac impairment, and higher NT-proBNP, sST2, and hs-TnT concentrations [115]. In fact, circulating IL- $1 \beta$ levels had been clinically meaningful in HF patients interfering with the predictive ability of sST2. Indeed, regardless of LVEF, HF patients with low sST2 $(\geq 35.0 \mathrm{ng} / \mathrm{ml})$ and also low IL- $1 \beta$ $(\geq 49.1 \mathrm{pg} / \mathrm{ml})$ had significantly lower risk of CV death, HF-related outcomes including readmission, than among patients with high sST2 $(>35.0 \mathrm{ng} / \mathrm{ml})$ and also high IL$1 \beta(<49.1 \mathrm{pg} / \mathrm{ml})$ levels [115].

6.2. Soluble Suppression of Tumorigenicity-2. Serum levels of IL-33 and soluble suppression of tumorigenicity-2 (sST2), which is the soluble form of IL-1 receptor-like 1 (IL-33), were significantly higher in HF regardless of the presence HF phenotypes associated with HF symptom severity, LV hypertrophy, and the risk of CV death and hospitalization than in healthy volunteers $[118,119]$. It was found that IL-33 improved cell viability after ischemia injury through ST2 signalling and suppression nuclear factor kappa-B that unleashed the upexpression of the antiapoptotic factors (XIAP, cIAP1, surviving) and HIF-1, preventing apoptosis [120]. In patients with AMI, serum levels of sST2 were found to be increased, and after adjustment for comorbidities, the Killip class and troponin T sST2 independently predicted the excess risk of death and HF [121]. Development of adverse cardiac remodelling due to AMI was strongly associated with the elevated levels of sST2 in the peripheral blood [122].

Serum sST2 served as a predictive biomarker in patients at risk of HF and in individuals with established chronic HF [123], but the prognostic value of the biomarker was diminished after adjusting for the clinical status including comorbidity presence (abdominal obesity, diabetes mellitus, and obstructive pulmonary disease) and NT-proBNP [124-126]. Additionally, sST2 was able to be helpful in short-term clinical outcome prognostication in acute HF and actually decompensated HF patients regardless of worsening kidney function, whereas renal failure was found to be a crucial factor for the NP predictive value $[127,128]$. In-patients survived after acute HF have yielded the concentrations of sST2 at discharge which were independently associated with sudden death, CV death, HF-related death, and HF readmission during the 3 -month period after discharge [127, 128]. Yet, sST2 yielded strong, independent predictive value for all-cause and cardiovascular mortality, and HF hospitalization in chronic HF, and deserves consideration to be part of a multimarker panel together with NT-proBNP and hsTnT [129]. The PARADIGM-HF trial (Prospective Comparison of ARNI With ACEI to Determine Impact on Global Mortality and Morbidity in Heart Failure) has revealed the levels of sST2 increased at 1 month which were associated with worse subsequent HF clinical outcomes, and the decreased SST2 concentrations were related to better prognosis particularly related to declined $\mathrm{CV}$ death and HF admission [130].

6.3. C-Reactive Protein. High-sensitive C-reactive protein (hs-CRP) has also markedly improved the risk stratification of acute HF and acutely decompensated HF patients in multibiomarker models, which predominantly included MRproADM and NT-proBNP [131, 132]. However, circulating levels of hs-CRP were associated with the New York Heart Association functional class of HF, primary hospitalizations and readmission predominantly in patients with HFrEF, but not HFpEF [133]. Unfortunately, hs-CRP did not add incremental value to NPs, sST2, and galectin-3 in patients with HFpEF rather than HFpEF $[133,134]$. The ASCENDHF trial has reported that the levels of hs-CRP at admission in acute HF patients were not associated with acute dyspnea improvement, in-hospital death, advancing HF, short-term (30 days) and long-term (180 days) mortality, and HF readmission $[135,136]$. On the contrary, at 30 days, elevated levels of hs-CRP among survivors were associated with higher 180-day mortality and readmission [135]. Although hs-CRP is under ongoing investigations, potential treatment options and goals of the therapy among HF individuals are not fully determined. 
6.4. Growth Differential Factor-15. Growth differentiation factor- (GDF-) 15 is determined as an inflammation and oxidative stress biomarker, which belongs to the TGF- $\beta$ cytokine superfamily and is highly expressed in myocardium and endothelial cells in CV disease including HF [137]. Previous studies have shown that GDF-15 protected the myocardium from ischemia and reperfusion injury [138, 139]. Higher serum levels of GDF-15 were associated with poor prognosis in acute $\mathrm{HF}$ independent from concentrations of NPs [140] and chronic HF irrespective of LVEF [141, 142]. Moreover, the Valsartan Heart Failure Trial has shown that serial measurements of GDF-15 had increased the incremental predictive power to the only measure at baseline for the severity of $\mathrm{HF}$ and prognosis [143]. Additionally, the elevated serum level of GDF-15 was the most prognostic biomarker in comparison to NT-proBNP, hs-CRP, and hs-TnT, in predicting long-term mortality in advanced HF [144]. Overall, a multimarker model based on NT-proBNP, hs-CRP, GDF-15, and hs-TnT had more predictable HFrEF and HFpEF than the isolating biomarker $[145,146]$. Probably, inflammatory mediators, such as sST2 and GDF-15, as it is expecting, can become molecular targets not only for the diagnosis but also for the treatment of adverse cardiac remodelling in the future.

\section{Biomarkers of Cardiac Fibrosis}

7.1. Galectin-3. Over the last decade, galectin-3 had been widely investigated as a biomarker of fibrosis and inflammation with a promising predictive value for HF development and $\mathrm{CV}$ events [147]. Galectin-3 is multifunction $\beta$-galactoside-binding protein, which belongs to lectin family and is expressed in several tissues and circulating cells, such as mononuclears, macrophages, progenitor cells, mast cells, and neutrophils [148]. Galectin-3 plays a pivotal role in inflammation, fibrosis, immunity, tissue repair, and cardiac remodelling and acts as a mediator of the development and progression of the diseases, for which these pathogenetic stages are crucial $[149,150]$. Indeed, galectin-3 is expressed in myocardium releasing from activated macrophages and contributes cardiac dysfunction through the remodelling of ECM and accumulation of collagen [151]. Additionally, galectin-3 is able to mediate cardiac and vascular fibrosis induced by overexpressed aldosterone [152]. However, there is evidence confirming the role of polymorphism of galectin3 gene in susceptibility to cardiac injury and fibrosis [153]. Being a mediator of both mutual relating processes-inflammation and fibrosis-galectin-3 was approved by the Food and Drugs Administration (USA) as a predictive biomarker for $\mathrm{HF}$ development and progression $[87,154]$. In fact, elevated levels of galectin-3 were found in patients with adverse cardiac remodelling regardless of HF phenotypes and it ethnologies [155, 156]. Therefore, galectin-3 having some advantages to NPs (more stability and resistance against hemodynamic overload and unloading state) predicted CV mortality and rehospitalization in HFrEF and HFpEF [157, 158]. Moreover, the TRIUMPH (Translational Initiative on Unique and Novel Strategies for Management of Patients with Heart Failure) has shown that repeated measures of serum levels of galectin-3 could be useful in routine clinical practice for HF prognostication and treatment monitoring [159]. However, head-to-head comparison of sST2 and galectin 3 has revealed the superiority of sST2 in long-term risk stratification in an ambulatory stable HF [160]. For future direction, these facts require to be investigated in detail in large clinical trials with large sample size, because a metaanalysis of a discriminative value of galectin-3 did not yield a confirmation of previously received data [161].

7.2. Biomarkers of Collagen Turnover. It has been postulated that biomarkers of collagen turnover, such as carboxyterminal telopeptide of collagen type I, amino-terminal propeptide of type III procollagen, MMPs, and tissue inhibitors of MMPs, may be useful for risk stratification of cardiac remodelling associated with $\mathrm{HFpEF}$ and $\mathrm{HFrEF}[162,163]$. Indeed, myocardial fibrosis being a major cause of diastolic dysfunction contributes predominantly to the HFpEF [164]. The ECM rearrangement corresponds to an intensity of the inflammation in myocardium, and serum levels of biomarkers of collagen turnover are mediated by a balance between degradation of ECM components and synthesis. Proliferative phase complimented to myocardial fibrosis is considered a typical response during late adverse cardiac remodelling, whereas increased degradation of ECM is suitable for AMI and early cardiac dilatation [165]. In fact, MMP-2, MMP-9, carboxytelopeptides of procollagen type I, and aminopeptide of procollagen type III had a predictive value for HFpEF that was equal NT-proBNP [163], while discriminative ability of elevated serum levels of MMP-2 was superior to NT-proBNP for early $\operatorname{HFpEF}[162,163,166]$. Whether emerging biomarkers of ECM rearrangement and collagen turnover is essential to identify asymptomatic patients with HFpEF after AMI with subsequent PCI is not fully clear, while a loss of myocardial collagen scaffolding plays a pivotal role in adverse cardiac remodelling with poor prognosis. Interestingly, elevated levels of C-terminal telopeptide were associated with global LVEF, the risk of $\mathrm{CV}$ death, and newly diagnosed or worsening HF due to various causes [167-169]. In this context, integrity of ECM biomarkers into personifying predictive strategy in AMI patients appears to be promised, because multiple biomarkers' approach with traditional biomarkers and indicators of ECM turnover may have increased the sensitivity and specificity of clinical outcomes in patients with adverse cardiac remodelling and isolated diastolic dysfunction.

\section{Biomarkers of Biomechanical Myocardial Stress}

8.1. Natriuretic Peptides. The physiologically natriuretic peptide (NP) system mediates water and sodium homeostasis playing a pivotal role in blood pressure enhancement, fluid retention, vascular function, structure remodelling of the heart, kidney, and vessels, and maintaining differentiation and repair tissue, and supports immunity, metabolic response, and inflammation [170]. There are at least four members of NP system, such as atrial NP (ANP), brain NP (BNP), C-type of NP, and D-type of NP [171]. Biological 
TABLE 2: CV and non-CV causes of elevating NPs in peripheral blood.

\begin{tabular}{lc}
\hline CV causes & Non-CV causes \\
\hline Acute and chronic HF & Sepsis/shock \\
LV hypertrophy & Severe infections \\
Pulmonary hypertension & Critical ill patients \\
ACS/AMI & Acute and chronic kidney failure \\
Stable CAD & Severe trauma/surgery \\
Multifocal atherosclerosis & Chronic obstructive pulmonary \\
Cardiomyopathies & disease \\
Myocarditis & Severe bronchial asthma \\
Atrial fibrillation and flutter & Pneumonia \\
Hypertension & Large burns and frostbite \\
Congenital and acquired & Stroke \\
valvular heart disease & Kidney amyloidosis \\
Pericardial disease & Diabetes mellitus \\
Cardiac toxicity due to & Thyroid dysfunction \\
tumoricidal therapy & Anemia \\
Electrical & Pleural disease \\
Successful resuscitation &
\end{tabular}

HF: heart failure; LV: left ventricular; ACS: acute coronary syndrome; AMI: acute myocardial infarction; CAD: coronary artery disease.

effects of NPs are provided through interacting with appropriate receptors: NPR-A, NPR-B, and NPR-C. Kidney effects of NPs are diuresis and wateresis due to the decreasing tubular reabsorption of sodium and water, increasing glomerular filtration rate (GFR) in result of inducing afferent arteriole vasodilation, and protection of the kidney from metabolic and ischemia injury [172]. Vascular effects of NPs correspond to vasodilation, support, capillary permeability and vascular reparation, and antiproliferative and hypocoagulative effects [173]. NPs mediate cardiac protection with respect to decreasing preload and afterload, diminishing biomechanical stress, and maintaining anti-ischemic, antiproliferative, and antiapoptotic abilities. Therefore, NPs have direct inotropic and antiarrhythmic effects [174]. Overall, the NP system is a physiological antagonist of RAAS and the sympathoadrenal system. The main triggers for synthesis and release of NPs are myocardial stretching, fluid retention, increase of pre- and postload, BP elevation, decreasing GFR, and ischemia of target organs (kidney, heart, and brain). Therefore, adipocytes and glial cells can produce NPs as a result of proinflammatory stimulation [175].

Increased activity of a circulating and local NP system was determined in patients with CV disease including LV hypertrophy, AMI, stable coronary artery disease, hypertension, and HF [176]. However, there are large numbers of causes distinguishing from $\mathrm{CV}$ and accompanying elevation of circulating levels of NPs (see Table 2). There is a large body of evidence showing that NP production occurs in close relation to the severity of LV systolic dysfunction, and the circulating levels of BNP and ANP strongly correspond to the New York Heart Association functional class of HF [88].
However, the production of NPs in advanced HF became blunt and irrespective of how high concentration of NPs in peripheral blood fluid retention, vasoconstriction, and cardiac dysfunction appears to progressed. In contrast, adequate treatment of HF, which is associated with improvement of clinical status and increase of tolerance to physical exercise, corresponds to declining circulating levels of BNP and ANP [177].

Therefore, patients with abdominal obesity frequently present less levels of BNP that it is expected due to increased circulating levels of neprilysin, which degradates BNP [178]. Although older age and female sex are the most common reason association with increased levels of NPs in circulation beyond relative causes, some structural abnormalities corresponding to decreased mean $e^{\prime}$ velocity and increased mitral early flow velocity/early diastolic tissue velocity ratio can be found [179-181].

Current clinical recommendations are considered NPs predominantly BNP, NT-proBNP, and NT-proANP, as diagnostic and predictive biomarkers for HF regardless of LVEF, as well as a tool for risk stratification in general population $[86,87]$. However, elevated levels of NPs $(B N P \geq 100 \mathrm{pg} / \mathrm{ml}$ or NT - proBNP $\geq 300 \mathrm{pg} / \mathrm{ml}$; or $\mathrm{BNP} \geq 300 \mathrm{pg} / \mathrm{ml}$ or NT proBNP $\geq 900 \mathrm{pg} / \mathrm{ml}$ if in atrial fibrillation/flutter) in patients with suspected HFmrEF/HFpEF were found to confirm the diagnosis [182]. NPs are also excellent prognostic biomarkers of adverse cardiac remodelling after AMI, whereas the clinical value of such discriminative ability is less clear than established acute and chronic HF [183]. Therefore, decreased levels of NT - proBNP $<1000 \mathrm{pg} / \mathrm{ml}$ as a result of HF therapy was associated with lower 180-day mortality and readmission in comparison with $\mathrm{NT}-$ proBNP $\geq 1000 \mathrm{pg} / \mathrm{ml}$, whereas NT-proBNP reduction of $>30 \%$ from initial levels did not improve 6-month outcomes and was not more effective than a traditional treatment [184-186]. Overall, elevated levels of NPs including NT-proBNP and NT-proANP had higher negative diagnostic value than the positive diagnostic value for HF, while the positive predictive ability of NPs in elevating concentrations was superior to the negative predictive value for asymptomatic cardiac remodelling, as well as HF regardless of LVEF. In fact, high individual variability, depending on the serum levels of NPs on comorbidities, including GFR, abdominal obesity, and older age and female sex, gives more opportunities to rule out major structural cardiac abnormalities and HF, when NP levels are normal or near normal. Confirmation of the HF and cardiac remodelling with isolating diastolic dysfunction requires more predictive information including clinical conditions, diastolic characteristics, measure of LVEF, and other biomarker assay.

8.2. Copeptin. Copeptin is a stable 39 -aminoacid glycopeptide derived from C-terminal portion of the precursor of arginine vasopressin, which is a key regulator of water homeostasis and plasma osmolality [187]. Serum levels of copeptin have exhibited close linear correlation with concentrations of arginine vasopressin and are use as surrogate biomarker of its secretion [188]. There is evidence that elevated serum levels of copeptin are a diagnostic biomarker of 
asymptomatic cardiac remodelling, HF, sepsis, acute kidney injury, insulin resistance, and metabolic syndrome [189]. Several trials have yielded that increased levels of copeptin were strong predictor of mortality in patients with acute and chronic HF $[189,190]$, stroke [191], end stage of renal disease [192], stable CAD [193], and diabetes mellitus [194]. However, there is a large number of confounding factors (hydration status, gender, blood pressure, GFR, and body mass), which make it difficult to interpret data of copeptin levels in patients with known CV disease, as well as in healthy individuals [195]. Additionally, copeptin was not better than the NPs in the diagnosis and prognosis of $\mathrm{HF}$ as well as in prognostication of adverse cardiac remodelling after AMI [196].

8.3. Midregional Proadrenomedullin. Midregional proadrenomedullin (MR-proADM) is stable peptide fragment that is precursor for adrenomedullin (ADM) and generated through posttranslational processing from preproadrenomedullin [197]. ADM is expressed in several tissues (adrenal medulla, brain, kidney, lung, spleen, liver, and vasculature) and cells (endothelial cells, cardiac myocytes, vascular smooth muscle cells, and epithelial cells) and mediates natriuresis, diuresis, vasodilation, positive inotropic effect, and hypotension [198].

Early clinical trials have shown that circulating levels of MR-proADM were significantly increased in patients with acute HF and STEMI [199, 200], and a cut-off value of $0.79 \mathrm{nmol} / \mathrm{l}$ has been yielded to be associated with adverse outcomes including death [201, 202]. Additionally, serum levels of MR-proADM $>0.70 \mathrm{nmol} / \mathrm{l}$ were proposed to be the rule-in criteria of AMI [203].

The MR-proADM has become a biomarker that was specifically investigated as a possible prognosticator of acute HF and early outcomes in STEMI patients undergoing PCI. The BACH (Biomarkers in Acute Heart Failure) study revealed that increased serum levels of MRproANP were a useful diagnostic biomarker as BNP for acute HF in patients with acute dyspnoe [204]. The results of the DANAMI-3 (The Danish Study of Optimal Acute Treatment of Patients with ST-segment-elevation myocardial infarction) study have shown that elevated levels of MR-proADM were strong predictor of shortand long-term mortality and hospital admission for $\mathrm{HF}$ after AMI [205]. Unfortunately, MR-proADM has demonstrated predictive ability with high similarity to BNP, MR-proANP, and copeptin for one-year all-cause mortality in acute HF [206]. However, the measure of MRproADM may give additional diagnostic and prognostic information for incident $\mathrm{CV}$ events associated with advanced atherosclerosis that is useful for risk stratification among patients with adverse cardiac remodelling after AMI with subsequent PCI [207, 208]. Therefore, MR-proADM was able to predict major adverse cardiac events in patients suspecting AMI regardless of $\mathrm{HF}$ [209]. Moreover, in contrast to NPs, MR-proADM did not exhibit lowered concentration in obese patients with known HF that may facilitate diagnosis and prognosis of $\mathrm{HF}$ in this patient population [210].

\section{Other Biomarkers of Cardiac Remodelling}

9.1. Noncoding RNAs. Noncoding RNAs are powerful epigenetic regulators of cardiac gene expression and mediators of cardiac homeostasis and functions [211]. There are several types of noncoding RNAs, such as microRNAs (miRNAs), long noncoding RNAs, and circular RNAs, which play a central role in the regulation of numerous pathogenetic mechanisms and coordinate coupling of morbidity state with susceptibility to inflammatory and proliferative response [212]. Among these types of noncoding RNAs, various miRNAs are widely investigated (see Figure 4). Although there is a large body of evidence regarding up- and downregulation of genes for potassium channels, SERCA, subunits of receptors, signal molecules, proinflammatory cytokines, apoptotic mediators (Bax, caspase-9) in myocardium [213-216], and miRNAs are considered rather targets for personifying intervention and translational therapy, as well as prognosticators than diagnostic biomarkers for adverse cardiac remodelling and HF [217]. However, having signatures of miRNAs, which correspond to adverse cardiac remodelling, HF, sudden death, and cardiac abnormalities with established poor prognosis, such as concentric LV hypertrophy, fibrosis, and inflammation, it has not completely understood whether the "miRNA card" personally created for each patient will have clinical significance in the prediction of $\operatorname{HF}[33,218]$.

9.2. Circulating Mononuclear and Endothelial Progenitor Cells. Mononuclears (MPCs) and endothelial progenitor cells (EPCs) are essential components of endogenous vascular repair system that is activated as a result of several triggers, such as ischemia/hypoxia, inflammation, shear stress, thrombosis, infiltration of lipids, direct injury of vasculature, and endothelium [32].

It has been hypothesized that mobilization of MPCs/EPCs and increase in growth and differentiation into mature cells in vasculature accompany acute events, including AMI and acute HF, and are associated with vascular reparation $[219,220]$. However, previous acute CV events and chronic metabolic and CV diseases, such as diabetes mellitus, abdominal obesity, and hypertension, were reported to be causes of an exhausting pool of circulating angiopoetic MPCs/EPCs with immune phenotypes CD45+CD34+, CD45+CD34+CD133+, and CD45+CD34+CD133+CD184+ [221]. Consequently, advanced $\mathrm{HF}$ and progression of AMI-induced adverse cardiac remodelling were related to impaired vascular repair, vascularization, and angiogenesis due to a declined number of circulating precursors and lowered their function and survival [222]. This phenomenon is known as progenitor cell dysfunction and considered a promising predictive biomarker for $\mathrm{CV}$ mortality and $\mathrm{HF}$ progression and admission [223, 224], as well as in patient population with AMI submitted to PCI [225]. Probably, coronary circulating proangiogenic MPCs/EPCs collected from coronary sinus in AMI patients with subsequent PCI can become a powerful biomarker with increased accuracy in the prediction of adverse cardiac remodelling. However, the number and functionality of proangiogenic circulating precursors appear as promising biomarkers for the 


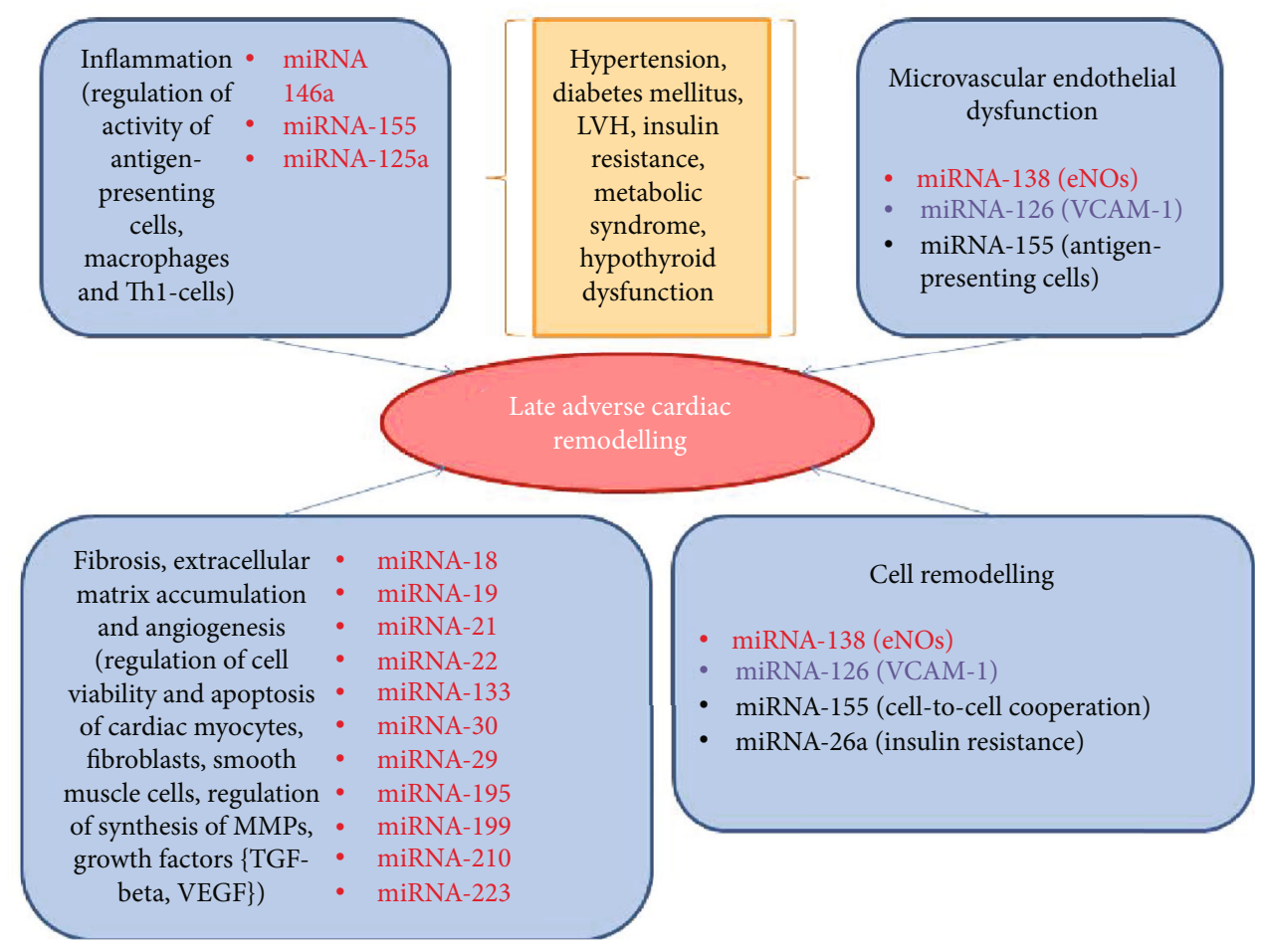

FIGURE 4: The role of miRNAs in the pathogenesis of late adverse cardiac remodelling in AMI. VEGF: vascular endothelial growth factor; TGF: transforming growth factor; NO: nitric oxide; eNOs: endothelial NO synthase; MMP: matrix metalloproteinase; VCAM: vascular adhesive molecule.

prediction of cardiac remodelling and HF development. Large clinical trials are required to clearly understand the role of new biomarkers in the diagnostic and predictive strategies among AMI patients with PCI.

9.3. Future Perspectives. There are numerous biomarkers, which were investigated as candidates for risk stratification and prognosis in AMI patients with PCI, such as activated and apoptotic endothelial cell-derived microvesicles, bone-related proteins (osteopontin, osteoprotegerin, and osteonectin), adipokines, gastrointestinal hormones, apelin, cardiotrophin-1, defensin-1 and defensin-2, macrophage inhibitory cytokine-1, circular RNAs, and gene card. Although the data received appear to be promising, there is no clear understanding whether diagnostic and predictive abilities of these biomarkers will be better than the conventional biomarkers of biomechanical stress, inflammation, fibrosis, and cardiac injury.

\section{Conclusions}

Circulating biomarkers are a promising tool to stratify AMI patients undergoing PCI at high risk of poor cardiac recovery and HF development. NPs are traditionally recommended as diagnostic and predictive biomarkers for acute $\mathrm{HF}$ and chronic HF regardless of LVEF, whereas sST2, galectin-3, and cardiac troponins can be used optionally. Previous clinical studies have yielded that multimarker models, which were based on the combination of biomarkers of several pathological axes involved in the nature evolution of adverse cardiac remodelling (biomechanical myocardial stress, necrosis and injury of cardiac myocytes, and inflammation), have provided incremental prognostic information for prediction of CV death or HF in AMI patients with subsequent PCI. Future clinical trials with larger sample sizes are required to elucidate the role of personifying biomarker-based strategy for diagnostic, prediction, and treatment among patients suspecting adverse cardiac remodelling and HF.

\section{Abbreviations}

ACE: $\quad$ Angiotensin-converting enzyme

ADAMTS: A Disintegrin and Metalloproteinase with Thrombospondin motifs

ADM: adrenomedullin

AMI: $\quad$ Acute myocardial infarction

Apaf-1: $\quad$ Adaptor protein apoptotic protease activating factor 1

ARBs: Angiotensin-II receptor antagonists

Bcl-2: $\quad$ B-cell lymphoma 2

BES: Biolimus eluting stent

BMS: $\quad$ Bare metal stent

CRP: $\quad$ C-reactive protein

CV: $\quad$ Cardiovascular

DAMPs: Damage-Associated Molecular Patterns

DAPT: Dual antiplatelet therapy

DNA: Deoxyribonucleic acid

ECM: $\quad$ Extracellular matrix

EF: $\quad$ Ejection fraction

GDF15: Growth/differential factor-15 


$\begin{array}{ll}\mathrm{H}_{2} \mathrm{O}_{2}: & \text { Hydrogen peroxide } \\ \text { HF: } & \text { Heart failure } \\ \text { HFmrEF: } & \text { Heart failure with midrange ejection fraction } \\ \text { HFpEF: } & \text { Heart failure with preserved ejection fraction } \\ \text { HFrEF: } & \text { Heart failure with reduced ejection fraction } \\ \text { HIF: } & \text { Hypoxia-induce factor } \\ \text { HMGB1: } & \text { High-mobility group 1B protein } \\ \text { HO-1: } & \text { Haem oxygenase-1 } \\ \text { HSP: } & \text { Heat shock proteins } \\ \text { IC: } & \text { Intracoronary } \\ \text { IL: } & \text { Interleukin } \\ \text { IVUS-VH: } & \text { Intravascular ultrasound virtual-histology } \\ \text { LncRNA: } & \text { Long noncoding RNA } \\ \text { LV: } & \text { Left ventricle } \\ \text { MAPK: } & \text { Mitogen-activated protein kinase } \\ \text { MCRA: } & \text { Mineralocorticoid receptor antagonists } \\ \text { miRNA: } & \text { MicroRNA } \\ \text { MMP: } & \text { Matrix metalloproteinase } \\ \text { NO: } & \text { Nitric oxide } \\ \text { NPs: } & \text { Natriuretic peptides } \\ \text { OCT: } & \text { Optical coherence tomography } \\ \text { PCI: } & \text { Percutaneous coronary intervention } \\ \text { RAAS: } & \text { Renin-angiotensin-aldosterone system } \\ \text { RNA: } & \text { Ribonucleic acid } \\ \text { ROS: } & \text { Reactive oxygen species } \\ \text { SOD: } & \text { Superoxide dismutase } \\ \text { sST2: } & \text { Soluble suppression of tumorigenicity-2 } \\ \text { STEMI: } & \text { ST-segment elevation myocardial infarction } \\ \text { TIMI score: } & \text { Thrombolysis in Myocardial Infarction score } \\ \text { TGF: } & \text { Transforming growth factor } \\ \text { TLR: } & \text { Toll-like receptor } \\ \text { TNF: } & \text { Tumor necrosis factor } \\ \text { TSP: } & \text { Thrombospondin } \\ \text { VEGF: } & \text { Vascular endothelial growth factor. } \\ & \end{array}$

\section{Data Availability}

This is a narrative review, so dataset was not created.

\section{Conflicts of Interest}

The authors declare that there is no conflict of interest regarding the publication of this paper.

\section{References}

[1] H. E. Carter, D. Schofield, and R. Shrestha, "Productivity costs of cardiovascular disease mortality across disease types and socioeconomic groups," Open Heart, vol. 6, no. 1, article e000939, 2019.

[2] J. Yap, S. Y. Chia, F. Y. Lim et al., "The Singapore Heart Failure Risk Score: prediction of survival in Southeast Asian patients," Annals of the Academy of Medicine of Singapore, vol. 48, no. 3, pp. 86-94, 2019.

[3] X. Chen, G. Savarese, U. Dahlström, L. H. Lund, and M. Fu, "Age-dependent differences in clinical phenotype and prognosis in heart failure with mid-range ejection compared with heart failure with reduced or preserved ejection fraction," Clinical Research in Cardiology, vol. 108, no. 12, pp. 13941405, 2019, [Epub ahead of print].
[4] P. M. Seferović, M. Polovina, J. Bauersachs et al., "Heart failure in cardiomyopathies: a position paper from the Heart Failure Association of the European Society of Cardiology," European Journal of Heart Failure, vol. 21, no. 5, pp. 553576, 2019.

[5] K. Dharmarajan and M. W. Rich, "Epidemiology, pathophysiology, and prognosis of heart failure in older adults," Heart Failure Clinics, vol. 13, no. 3, pp. 417-426, 2017.

[6] A. Slee, M. Saad, and S. Saksena, "Heart failure progression and mortality in atrial fibrillation patients with preserved or reduced left ventricular ejection fraction," Journal of Interventional Cardiac Electrophysiology, vol. 55, no. 3, pp. 325331, 2019, [Epub ahead of print].

[7] Y. Sato, A. Yoshihisa, M. Oikawa et al., "Prognostic Impact of Worsening Renal Function in Hospitalized Heart Failure Patients With Preserved Ejection Fraction: A Report From the JASPER Registry," Journal of Cardiac Failure, vol. 25, no. 8, pp. 631-642, 2019, [Epub ahead of print].

[8] M. Nakamura and J. Sadoshima, "Cardiomyopathy in obesity, insulin resistance and diabetes," The Journal of Physiology, 2019, [Epub ahead of print].

[9] L. Shen, P. S. Jhund, K. F. Docherty et al., "Prior pacemaker implantation and clinical outcomes in patients with heart failure and preserved ejection fraction," JACC: Heart Failure, vol. 7, no. 5, pp. 418-427, 2019, [Epub ahead of print].

[10] H. Seligman, M. J. Shun-Shin, A. Vasireddy et al., "Fractional flow reserve derived from microcatheters versus standard pressure wires: a stenosis-level meta-analysis," Open Heart, vol. 6, no. 1, article e000971, 2019.

[11] K. V. Patel, R. Mauricio, J. L. Grodin et al., "Identifying a lowflow phenotype in heart failure with preserved ejection fraction: a secondary analysis of the RELAX trial," ESC Heart Failure, vol. 6, no. 4, pp. 613-620, 2019.

[12] G. V. Halade, V. Kain, B. Tourki, and J. K. Jadapalli, "Lipoxygenase drives lipidomic and metabolic reprogramming in ischemic heart failure," Metabolism, vol. 96, pp. 22-32, 2019, [Epub ahead of print].

[13] J. P. Tsai, K. T. Sung, C. H. Su et al., "Diagnostic accuracy of left atrial remodelling and natriuretic peptide levels for preclinical heart failure," ESC Heart Failure, vol. 6, no. 4, pp. 723-732, 2019, [Epub ahead of print].

[14] A. Burlacu, P. Simion, I. Nistor, A. Covic, and G. Tinica, "Novel percutaneous interventional therapies in heart failure with preserved ejection fraction: an integrative review," Heart Failure Reviews, vol. 24, no. 5, pp. 793-803, 2019, [Epub ahead of print].

[15] H. Zeng and J. X. Chen, "Microvascular rarefaction and heart failure with preserved ejection fraction," Frontiers in Cardiovascular Medicine, vol. 6, 2019.

[16] R. Scarsini, G. L. de Maria, A. Borlotti et al., "Incremental value of coronary microcirculation resistive reserve ratio in predicting the extent of myocardial infarction in patients with STEMI. Insights from the Oxford Acute Myocardial Infarction (OxAMI) study," Cardiovascular Revascularization Medicine, vol. 20, no. 12, pp. 1148-1155, 2019, [Epub ahead of print].

[17] B. Ky, B. French, A. May Khan et al., "Ventricular-Arterial Coupling, Remodeling, and Prognosis in Chronic Heart Failure," Journal of the American College of Cardiology, vol. 62, no. 13, pp. 1165-1172, 2013.

[18] B. He, L. Gai, J. Gai et al., "Correlation between major adverse cardiac events and coronary plaque characteristics," 
Experimental \& Clinical Cardiology, vol. 18, no. 2, pp. e71e76, 2013.

[19] A. S. Bhatt, A. P. Ambrosy, and E. J. Velazquez, "Adverse remodeling and reverse remodeling after myocardial infarction," Current Cardiology Reports, vol. 19, no. 8, 2017.

[20] A. S. Blom, J. J. Pilla, R. C. Gorman III et al., "Infarct size reduction and attenuation of global left ventricular remodeling with the CorCap cardiac support device following acute myocardial infarction in sheep," Heart Failure Reviews, vol. 10, no. 2, pp. 125-139, 2005.

[21] A. W. Schoenenberger, P. Jamshidi, R. Kobza et al., "Progression of coronary artery disease during long-term follow-up of the Swiss Interventional Study on Silent Ischemia Type II (SWISSI II)," Clinical Cardiology, vol. 33, no. 5, pp. 289295, 2010.

[22] P. Erne, A. W. Schoenenberger, D. Burckhardt et al., "Effects of percutaneous coronary interventions in silent ischemia after myocardial infarction: the SWISSI II randomized controlled trial," JAMA, vol. 297, no. 18, pp. 1985-1991, 2007.

[23] G. A. Sgueglia, F. D'Errico, G. Gioffrè et al., "Angiographic and clinical performance of polymer-free biolimus-eluting stent in patients with ST-segment elevation acute myocardial infarction in a metropolitan public hospital: the BESAMI MUCHO study," Catheterization and Cardiovascular Interventions, vol. 91, no. 5, pp. 851-858, 2018.

[24] C. R. Jones, S. J. Khandhar, M. Ramratnam et al., "Identification of intrastent pathology associated with late stent thrombosis using optical coherence tomography," Journal of Interventional Cardiology, vol. 28, no. 5, pp. 439-448, 2015.

[25] A. Al Mamary, G. Dariol, and M. Napodano, "Late stent fracture - A potential role of left ventricular dilatation," Journal of the Saudi Heart Association, vol. 26, no. 3, pp. 162-165, 2014.

[26] S. V. Rao, U. Zeymer, P. S. Douglas et al., "A randomized, double-blind, placebo-controlled trial to evaluate the safety and effectiveness of intracoronary application of a novel bioabsorbable cardiac matrix for the prevention of ventricular remodeling after large ST- segment elevation myocardial infarction: Rationale and design of the PRESERVATION I trial," American Heart Journal, vol. 170, no. 5, pp. 929-937, 2015, Epub 2015 Aug 24.

[27] S. V. Rao, U. Zeymer, P. S. Douglas et al., "Bioabsorbable intracoronary matrix for prevention of ventricular remodeling after myocardial infarction," Journal of the American College of Cardiology, vol. 68, no. 7, pp. 715-723, 2016.

[28] T. Mizoguchi, T. Sawada, T. Shinke et al., "Detailed comparison of intra-stent conditions 12 months after implantation of everolimus-eluting stents in patients with ST-segment elevation myocardial infarction or stable angina pectoris," International Journal of Cardiology, vol. 171, no. 2, pp. 224-230, 2014, Epub 2013 Dec 21.

[29] S. H. Rezkalla, R. V. Stankowski, J. Hanna, and R. A. Kloner, "Management of no-reflow phenomenon in the catheterization laboratory," JACC: Cardiovascular Interventions, vol. 10, no. 3, pp. 215-223, 2017.

[30] J. Barrabes, "Comments on the 2015 ESC Guidelines for the Management of Acute Coronary Syndromes in Patients Presenting Without Persistent ST-segment Elevation," Revista Española de Cardiología (English Edition), vol. 68, no. 12, pp. 1061-1067, 2015.

[31] T. K. Steigen, C. E. Buller, G. B. John Mancini et al., "Myocardial perfusion grade after late infarct artery recanalization is associated with global and regional left ventricular function at one year: analysis from the Total Occlusion Study of Canada-2," Circulation: Cardiovascular Interventions, vol. 3, no. 6, pp. 549-555, 2010.

[32] A. E. Berezin, "Endogenous vascular repair system in cardiovascular disease: the role of endothelial progenitor cells," Australasian Medical Journal, vol. 12, no. 2, pp. 42-48, 2019.

[33] A. Berezin, "Epigenetics in heart failure phenotypes," $B B A$ Clinical, vol. 6, pp. 31-37, 2016.

[34] A. Celik, N. Kalay, H. Korkmaz et al., "Short-term left ventricular remodeling after revascularization in subacute total and subtotal occlusion with the infarct-related left anterior descending artery," Cardiology Research, vol. 2, no. 5, pp. 229-235, 2011.

[35] M. E. Pfusterer, P. Buser, S. Osswald, P. Weiss, J. Bremerich, and F. Burkart, "Time dependence of left ventricular recovery after delayed recanalization of an occluded infarct-related coronary artery: findings of a pilot study," Journal of the American College of Cardiology, vol. 32, no. 1, pp. 97-102, 1998.

[36] N. Reifart, "Challenges in complicated coronary chronic total occlusion recanalisation," Interventional Cardiology Review, vol. 8, no. 2, pp. 107-111, 2013.

[37] N. G. Frangogiannis, "Pathophysiology of myocardial infarction," Comprehensive Physiology, vol. 20, pp. 1841-1875, 2015.

[38] M. Neri, I. Riezzo, N. Pascale, C. Pomara, and E. Turillazzi, "Ischemia/reperfusion injury following acute myocardial infarction: a critical issue for clinicians and forensic pathologists," Mediators Inflamm, vol. 2017, article 7018393, 14 pages, 2017, Epub 2017 Feb 13.

[39] D. Y. Fuhrman and J. A. Kellum, "Remote ischemic preconditioning in the PICU: a simple concept with a complex past," Pediatric Critical Care Medicine, vol. 17, no. 8, pp. e371-e379, 2016.

[40] S. Hernandez-Resendiz, K. Chinda, S. B. Ong, H. CabreraFuentes, C. Zazueta, and D. Hausenloy, "The role of redox dysregulation in the inflammatory response to acute myocardial ischaemia-reperfusion injury - adding fuel to the fire," Current Medicinal Chemistry, vol. 25, no. 11, pp. 12751293, 2018.

[41] K. Przyklenk and P. Whittaker, "Remote ischemic preconditioning: current knowledge, unresolved questions, and future priorities," Journal of Cardiovascular Pharmacology and Therapeutics, vol. 16, no. 3-4, pp. 255-259, 2016.

[42] D. M. Yellon and D. J. Hausenloy, "Myocardial reperfusion injury," The New England Journal of Medicine, vol. 357, no. 11, pp. 1121-1135, 2007.

[43] P. Pagliaro, F. Moro, F. Tullio, M.-G. Perrelli, and C. Penna, "Cardioprotective pathways during reperfusion: focus on redox signaling and other modalities of cell Signaling," Antioxidants \& Redox Signaling, vol. 14, no. 5, pp. 833-850, 2011.

[44] B. Long, N. Li, X. X. Xu et al., "Long noncoding RNA FTX regulates cardiomyocyte apoptosis by targeting miR-29b-1$5 \mathrm{p}$ and Bcl212," Biochemical and Biophysical Research Communications, vol. 495, no. 1, pp. 312-318, 2018.

[45] T. Kalogeris, Y. Bao, and R. J. Korthuis, "Mitochondrial reactive oxygen species: a double edged sword in ischemia/reperfusion vs preconditioning," Redox Biology, vol. 2, no. 1, pp. 702-714, 2014. 
[46] N. Zhang, X. Meng, L. Mei, J. Hu, C. Zhao, and W. Chen, "The long non-coding RNA SNHG1 attenuates cell apoptosis by regulating miR-195 and BCL2-like protein 2 in human cardiomyocytes," Cellular Physiology and Biochemistry, vol. 50, no. 3, pp. 1029-1040, 2018.

[47] F. He, H. Liu, J. Guo et al., "Inhibition of microRNA-124 reduces cardiomyocyte apoptosis following myocardial infarction via targeting STAT3," Cellular Physiology and Biochemistry, vol. 51, no. 1, pp. 186-200, 2018.

[48] C. Sun, H. Liu, J. Guo et al., "MicroRNA-98 negatively regulates myocardial infarction-induced apoptosis by downregulating Fas and caspase-3," Scientific Reports, vol. 7, no. 1, p. 7460, 2017.

[49] T. Okamoto, T. Akaike, T. Sawa, Y. Miyamoto, A. Van der Vliet, and H. Maeda, "Activation of matrix metalloproteinases by peroxynitrite-induced protein S-glutathiolation via disulfide S-oxide formation," The Journal of Biological Chemistry, vol. 276, no. 31, pp. 29596-29602, 2001.

[50] P.-Y. Cheung, G. Sawicki, M. Wozniak, W. Wang, M. W. Radomski, and R. Schulz, "Matrix metalloproteinase-2 contributes to ischemia-reperfusion injury in the heart," Circulation, vol. 101, no. 15, pp. 1833-1839, 2000.

[51] A. E. Berezin and T. A. Samura, "Prognostic value of biological markers in myocardial infarction patients," Asian Cardiovascular and Thoracic Annals, vol. 21, no. 2, pp. 142-150, 2013.

[52] C. Fernandez-Patron, M. W. Radomski, and S. T. Davidge, "Vascular matrix metalloproteinase-2 cleaves big endothelin-1 yielding a novel vasoconstrictor," Circulation Research, vol. 85, no. 10, pp. 906-911, 1999.

[53] D. Edwards, M. Handsley, and C. Pennington, "The ADAM metalloproteinases," Molecular Aspects of Medicine, vol. 29, no. 5, pp. 258-289, 2008.

[54] O. V. Petyunina, M. P. Kopytsya, and A. E. Berezin, "Biomarker-based prognostication of adverse cardiac remodeling after STEMI: the role of single nucleotide polymorphism T786C in endothelial NO-synthase gene," Journal of Cardiology and Therapy, vol. 6, no. 1, pp. 768-774, 2019.

[55] S. Cardin, M. P. Scott-Boyer, S. Praktiknjo et al., "Differences in cell-type-specific responses to angiotensin II explain cardiac remodeling differences in C57BL/6 mouse substrains," Hypertension, vol. 64, no. 5, pp. 1040-1046, 2014.

[56] Y. Li, X._. He, C. Li, L. Gong, and M. Liu, "Identification of candidate genes and microRNAs for acute myocardial infarction by weighted gene coexpression network analysis," BioMed Research International, vol. 2019, Article ID 5742608, 11 pages, 2019.

[57] L. M. Buja, "Myocardial ischemia and reperfusion injury," Cardiovascular Pathology, vol. 14, no. 4, pp. 170-175, 2005.

[58] C. P. Baines, "How and when do myocytes die during ischemia and reperfusion: the late phase," Journal of Cardiovascular Pharmacology and Therapeutics, vol. 16, no. 3-4, pp. 239243, 2016.

[59] Z. Zhao, "Oxidative stress-elicited myocardial apoptosis during reperfusion," Current Opinion in Pharmacology, vol. 4, no. 2, pp. 159-165, 2004.

[60] A. Prasad, G. W. Stone, D. R. Holmes, and B. Gersh, "Reperfusion injury, microvascular dysfunction, and cardioprotection: the 'dark side' of reperfusion," Circulation, vol. 120, no. 21, pp. 2105-2112, 2009.
[61] P. Ferdinandy, D. J. Hausenloy, G. Heusch, G. F. Baxter, and R. Schulz, "Interaction of risk factors, comorbidities, and comedications with ischemia/reperfusion injury and cardioprotection by preconditioning, postconditioning, and remote conditioning," Pharmacological Reviews, vol. 66, no. 4, pp. 1142-1174, 2014.

[62] R. S. Vander Heide and C. Steenbergen, "Cardioprotection and Myocardial Reperfusion," Circulation Research, vol. 113, no. 4, pp. 464-477, 2013.

[63] K. McCafferty, S. Forbes, C. Thiemermann, and M. M. Yaqoob, "The challenge of translating ischemic conditioning from animal models to humans: the role of comorbidities," Disease Models \& Mechanisms, vol. 7, no. 12, pp. 13211333, 2014.

[64] A. Bonaventura, F. Montecucco, F. Dallegri et al., "Novel findings in neutrophil biology and their impact on cardiovascular disease," Cardiovascular Research, vol. 115, no. 8, pp. 1266-1285, 2019, [Epub ahead of print].

[65] M. A. Razzaque, X. Xu, M. Han, A. Badami, and S. A. Akhter, "Inhibition of postinfarction ventricular remodeling by high molecular weight polyethylene glycol," Journal of Surgical Research, vol. 232, pp. 171-178, 2018.

[66] W. An, Y. Yu, Y. Zhang, Z. Zhang, Y. Yu, and X. Zhao, "Exogenous IL-19 attenuates acute ischaemic injury and improves survival in male mice with myocardial infarction," British Journal of Pharmacology, vol. 176, no. 5, pp. 699-710, 2019.

[67] M. Arita, T. Ohira, Y.-P. Sun, S. Elangovan, N. Chiang, and C. N. Serhan, "Resolvin E1 selectively interacts with leukotriene B4Receptor BLT1 and ChemR23 to regulate inflammation," The Journal of Immunology, vol. 178, no. 6, pp. 39123917, 2007.

[68] V. Kain, K. A. Ingle, R. A. Colas et al., "Resolvin D1 activates the inflammation resolving response at splenic and ventricular site following myocardial infarction leading to improved ventricular function," Journal of Molecular and Cellular Cardiology, vol. 84, pp. 24-35, 2015.

[69] L. A. Grisanti, T. P. Thomas, R. L. Carter et al., "Pepducinmediated cardioprotection via $\beta$-arrestin-biased $\beta 2$-adrenergic receptor-specific signaling," Theranostics, vol. 8 , no. 17 , pp. 4664-4678, 2018.

[70] Z. Guo, N. Liu, L. Chen, X. Zhao, and M. R. Li, "Independent roles of CGRP in cardioprotection and hemodynamic regulation in ischemic postconditioning," European Journal of Pharmacology, vol. 828, pp. 18-25, 2018.

[71] Q. Huang, Z. Yang, J. P. Zhou, and Y. Luo, "HMGB1 induces endothelial progenitor cells apoptosis via RAGE-dependent PERK/eIF2 $\alpha$ pathway," Molecular and Cellular Biochemistry, vol. 431, no. 1-2, pp. 67-74, 2017, Epub 2017 Mar 1.

[72] S. Frankenreiter, P. Bednarczyk, A. Kniess et al., "cGMP-elevating compounds and ischemic conditioning provide cardioprotection against ischemia and reperfusion injury via cardiomyocyte-specific BK channels," Circulation, vol. 136, no. 24, pp. 2337-2355, 2017.

[73] S. Raffa, X. L. D. Chin, R. Stanzione et al., "The reduction of NDUFC2 expression is associated with mitochondrial impairment in circulating mononuclear cells of patients with acute coronary syndrome," International Journal of Cardiology, vol. 286, pp. 127-133, 2019.

[74] J. Wang, M. Liu, Q. Wu et al., "Human embryonic stem cellderived cardiovascular progenitors repair infarcted hearts through modulation of MacrophagesviaActivation of signal transducer and activator of transcription 6," Antioxidants \& 
Redox Signaling, vol. 31, no. 5, pp. 369-386, 2019, [Epub ahead of print].

[75] Z. Zhong, J. Hou, Q. Zhang et al., "Differential expression of circulating long non-coding RNAs in patients with acute myocardial infarction," Medicine, vol. 97, no. 51, article e13066, 2018.

[76] Z. A. Ibrahim, C. L. Armour, S. Phipps, and M. B. Sukkar, "RAGE and TLRs: relatives, friends or neighbours?," Molecular Immunology, vol. 56, no. 4, pp. 739-744, 2013.

[77] J. J. De Haan, M. B. Smeets, G. Pasterkamp, and F. Arslan, "Danger signals in the initiation of the inflammatory response after myocardial infarction," Mediators of Inflammation, vol. 2013, Article ID 206039, 13 pages, 2013.

[78] A. Micera, B. O. Balzamino, A. D. Zazzo, F. Biamonte, G. Sica, and S. Bonini, "Toll-like receptors and tissue remodeling: the pro/cons recent findings," Journal of Cellular Physiology, vol. 231, no. 3, pp. 531-544, 2016.

[79] K. Fujiu, J. Wang, and R. Nagai, "Cardioprotective function of cardiac macrophages," Cardiovascular Research, vol. 102, no. 2, pp. 232-239, 2014.

[80] B. Tourki and G. Halade, "Leukocyte diversity in resolving and nonresolving mechanisms of cardiac remodeling," The FASEB Journal, vol. 31, no. 10, pp. 4226-4239, 2017.

[81] O. Dewald, P. Zymek, K. Winkelmann et al., "CCL2/monocyte chemoattractant protein-1 regulates inflammatory responses critical to healing myocardial infarcts," Circ. Res., vol. 96, no. 8, pp. 881-889, 2005.

[82] P. M. Henson, D. L. Bratton, and V. A. Fadok, "Apoptotic cell removal," Current Biology, vol. 11, no. 19, pp. R795-R805, 2001.

[83] S. Iravanian and S. C. Dudley Jr., "The renin-angiotensinaldosterone system (RAAS) and cardiac arrhythmias," Heart Rhythm, vol. 5, no. 6, pp. S12-S17, 2008.

[84] M. Massa, V. Rosti, M. Ferrario et al., "Increased circulating hematopoietic and endothelial progenitor cells in the early phase of acute myocardial infarction," Blood, vol. 105, no. 1, pp. 199-206, 2005.

[85] C. Gao, K. Howard-Quijano, C. Rau et al., "Inflammatory and apoptotic remodeling in autonomic nervous system following myocardial infarction," PLoS One, vol. 12, no. 5, article e0177750, 2017.

[86] P. Ponikowski, A. A. Voors, S. D. Anker et al., "2016 ESC guidelines for the diagnosis and treatment of acute and chronic heart failure: the task force for the diagnosis and treatment of acute and chronic heart failure of the European Society of Cardiology (ESC). Developed with the special contribution of the Heart Failure Association (HFA) of the ESC," European Journal of Heart Failure, vol. 18, no. 8, pp. 891-975, 2016.

[87] C. W. Yancy, M. Jessup, B. Bozkurt et al., "2017 ACC/AHA/HFSA focused update of the 2013 ACCF/AHA Guideline for the management of heart failure: a report of the American College of Cardiology/American Heart Association Task Force on Clinical Practice Guidelines and the Heart Failure Society of America," Circulation, vol. 136, no. 6, pp. e137e161, 2017.

[88] B. Bozkurt, "What is new in heart failure management in 2017? Update on ACC/AHA Heart Failure Guidelines," Current Cardiology Reports, vol. 20, no. 6, 2018.

[89] A. E. Berezin, "Circulating biomarkers in heart failure," Advances in Experimental Medicine and Biology, vol. 1067, pp. 89-108, 2018.
[90] A. E. Berezin, "Prognostication in different heart failure phenotypes: the role of circulating biomarkers," Journal of Circulating Biomarkers, vol. 5, no. 6, p. 6, 2016.

[91] M. Cheng, S. An, and J. Li, "Identifying key genes associated with acute myocardial infarction," Medicine, vol. 96, no. 42, article e7741, 2017.

[92] Y. Cao, R. Li, Y. Li et al., "Identification of transcription factor-gene regulatory network in acute myocardial infarction," Heart, Lung and Circulation, vol. 26, no. 4, pp. 343353, 2017, Epub 2016 Jul 26.

[93] B. Ibanez, S. James, S. Agewall et al., "2017 ESC Guidelines for the management of acute myocardial infarction in patients presenting with ST-segment elevation: the Task Force for the management of acute myocardial infarction in patients presenting with ST-segment elevation of the European Society of Cardiology (ESC)," European Heart Journal, vol. 39, no. 2, pp. 119-177, 2018.

[94] M. N. Lyngbakken, H. Røsjø, O. L. Holmen, H. Dalen, K. Hveem, and T. Omland, "Temporal changes in cardiac troponin I are associated with risk of cardiovascular events in the general population: the Nord-Trøndelag Health Study," Clinical Chemistry, vol. 65, no. 7, pp. 871-881, 2019, [Epub ahead of print].

[95] D. Soetkamp, K. Raedschelders, M. Mastali, K. Sobhani, C. N. Bairey Merz, and J. Van Eyk, "The continuing evolution of cardiac troponin I biomarker analysis: from protein to proteoform," Expert Review of Proteomics, vol. 14, no. 11, pp. 973-986, 2017.

[96] A. S. Shah, A. Anand, Y. Sandoval et al., "High-sensitivity cardiac troponin I at presentation in patients with suspected acute coronary syndrome: a cohort study," Lancet, vol. 386, no. 10012, pp. 2481-2488, 2015.

[97] D. Stelzle, A. S. V. Shah, A. Anand et al., "High-sensitivity cardiac troponin I and risk of heart failure in patients with suspected acute coronary syndrome: a cohort study," European Heart Journal - Quality of Care and Clinical Outcomes, vol. 4, no. 1, pp. 36-42, 2018.

[98] A. E. Berezin, "Circulating biomarkers in heart failure: diagnostic and prognostic importance," Journal of Laboratory and Precision Medicine, vol. 3, article 36, 2018.

[99] X. Jia, W. Sun, R. C. Hoogeveen et al., "High-Sensitivity Troponin I and Incident Coronary Events, Stroke, Heart Failure Hospitalization, and Mortality in the ARIC Study," Circulation, vol. 139, no. 23, pp. 2642-2653, 2019.

[100] P. Welsh, D. Preiss, C. Hayward et al., "Cardiac troponin $\mathrm{T}$ and troponin I in the general Population," Circulation, vol. 139, no. 24, pp. 2754-2764, 2019, [Epub ahead of print].

[101] T. Raskovalova, R. Twerenbold, P. O. Collinson et al., "Diagnostic accuracy of combined cardiac troponin and copeptin assessment for early rule-out of myocardial infarction: a systematic review and meta-analysis," European Heart Journal: Acute Cardiovascular Care, vol. 3, no. 1, pp. 18-27, 2014.

[102] J. Searle, O. Danne, C. Müller, and M. Mockel, "Biomarkers in acute coronary syndrome and percutaneous coronary intervention," Minerva Cardioangiologica, vol. 59, no. 3, pp. 203-223, 2011.

[103] C. Mueller, "Biomarkers and acute coronary syndromes: an update," European Heart Journal, vol. 35, no. 9, pp. 552556, 2014, Epub 2013 Dec 18. 
[104] M. Möckel and J. Searle, "Copeptin-marker of acute myocardial infarction," Current Atherosclerosis Reports, vol. 16, no. 7, p. 421, 2014.

[105] M. Karakas, J. L. Januzzi Jr., J. Meyer et al., "Copeptin does not add diagnostic information to high-sensitivity troponin $\mathrm{T}$ in low- to intermediate-risk patients with acute chest pain: results from the rule out myocardial infarction by computed tomography (ROMICAT) study," Clinical Chemistry, vol. 57, no. 8, pp. 1137-1145, 2011.

[106] S. Suzuki, H. Motoki, M. Minamisawa et al., "Prognostic significance of high-sensitivity cardiac troponin in patients with heart failure with preserved ejection fraction," Heart Vessels, vol. 34, no. 10, pp. 1650-1656, 2019, [Epub ahead of print].

[107] R. A. de Boer, M. Nayor, C. R. deFilippi et al., "Association of cardiovascular biomarkers with incident heart failure with preserved and reduced ejection fraction," JAMA Cardiology, vol. 3, no. 3, pp. 215-224, 2018.

[108] A. Gohar, J. P. C. Chong, O. W. Liew et al., "The prognostic value of highly sensitive cardiac troponin assays for adverse events in men and women with stable heart failure and a preserved vs. reduced ejection fraction," European Journal of Heart Failure, vol. 19, no. 12, pp. 1638-1647, 2017, Epub 2017 Aug 28.

[109] R. Santhanakrishnan, J. P. C. Chong, T. P. Ng et al., "Growth differentiation factor 15, ST2, high-sensitivity troponin T, and $\mathrm{N}$-terminal pro brain natriuretic peptide in heart failure with preserved vs. reduced ejection fraction," European Journal of Heart Failure, vol. 14, no. 12, pp. 1338-1347, 2012.

[110] P. Moliner, J. Lupón, J. Barallat et al., "Bio-profiling and bioprognostication of chronic heart failure with mid-range ejection fraction," International Journal of Cardiology, vol. 257, pp. 188-192, 2018.

[111] P. Welsh, L. Kou, C. Yu et al., "Prognostic importance of emerging cardiac, inflammatory, and renal biomarkers in chronic heart failure patients with reduced ejection fraction and anaemia: RED-HF study," European Journal of Heart Failure, vol. 20, no. 2, pp. 268-277, 2018.

[112] S. Sanders-van Wijk, V. van Empel, N. Davarzani et al., "Circulating biomarkers of distinct pathophysiological pathways in heart failure with preserved vs. reduced left ventricular ejection fraction," European Journal of Heart Failure, vol. 17, no. 10, pp. 1006-1014, 2015.

[113] S. L. Seliger, J. de Lemos, I. J. Neeland et al., "Older Adults, "Malignant" Left Ventricular Hypertrophy, and Associated Cardiac-Specific Biomarker Phenotypes to Identify the Differential Risk of New-Onset Reduced Versus Preserved Ejection Fraction Heart Failure: CHS (Cardiovascular Health Study)," JACC: Heart Failure, vol. 3, no. 6, pp. 445-455, 2015, Epub 2015 May 14.

[114] C. Sinning, T. Kempf, M. Schwarzl et al., "Biomarkers for characterization of heart failure - Distinction of heart failure with preserved and reduced ejection fraction," International Journal of Cardiology, vol. 227, pp. 272-277, 2017.

[115] D. A. Pascual-Figal, S. Manzano-Fernández, M. Boronat et al., "Soluble ST2, high-sensitivity troponin T- and Nterminal pro-B-type natriuretic peptide: complementary role for risk stratification in acutely decompensated heart failure," European Journal of Heart Failure, vol. 13, no. 7, pp. 718-725, 2011.

[116] D. A. Pascual-Figal, A. Bayes-Genis, M. C. Asensio-Lopez et al., "The interleukin-1 axis and risk of death in patients with acutely decompensated heart failure," Journal of the
American College of Cardiology, vol. 73, no. 9, pp. 10161025, 2019.

[117] Y. Cao, R. Li, F. Zhang, Z. Guo, S. Tuo, and Y. Li, "Correlation between angiopoietin-like proteins in inflammatory mediators in peripheral blood and severity of coronary arterial lesion in patients with acute myocardial infarction," Experimental and Therapeutic Medicine, vol. 17, no. 5, pp. 34953500, 2019.

[118] E. O. Weinberg, M. Shimpo, S. Hurwitz, S. Tominaga, J. L. Rouleau, and R. T. Lee, "Identification of serum soluble ST2 receptor as a novel heart failure biomarker," Circulation, vol. 107, no. 5, pp. 721-726, 2003.

[119] J. L. Januzzi, W. F. Peacock, A. S. Maisel et al., "Measurement of the Interleukin Family Member ST2 in Patients With Acute Dyspnea: Results From the PRIDE (Pro-Brain Natriuretic Peptide Investigation of Dyspnea in the Emergency Department) Study," Journal of the American College of Cardiology, vol. 50, no. 7, pp. 607-613, 2007.

[120] K. Seki, S. Sanada, A. Y. Kudinova et al., "Interleukin-33 prevents apoptosis and improves survival after experimental myocardial infarction through ST2 signaling," Circulation: Heart Failure, vol. 2, no. 6, pp. 684-691, 2009.

[121] W. S. Jenkins, V. L. Roger, A. S. Jaffe et al., "Prognostic value of soluble ST2 after myocardial infarction: a community perspective," The American Journal of Medicine, vol. 130, no. 9, pp. 1112.e9-1112.e15, 2017, Epub 2017 Mar 23.

[122] R. A. P. Weir, A. M. Miller, G. E. J. Murphy et al., "Serum soluble ST2: a potential novel mediator in left ventricular and infarct remodeling after acute myocardial infarction," Journal of the American College of Cardiology, vol. 55, no. 3, pp. 243250, 2010.

[123] W. H. W. Tang, Y. Wu, J. L. Grodin et al., "Prognostic value of baseline and changes in circulating soluble ST2 levels and the effects of nesiritide in acute decompensated heart failure," JACC: Heart Failure, vol. 4, no. 1, pp. 68-77, 2016.

[124] S. Manzano-Fernández, J. L. Januzzi, F. J. Pastor-Pérez et al., "Serial monitoring of soluble interleukin family member ST2 in patients with acutely decompensated heart failure," Cardiology, vol. 122, no. 3, pp. 158-166, 2012.

[125] T. Mueller, A. Gegenhuber, W. Poelz, and M. Haltmayer, "Diagnostic accuracy of B type natriuretic peptide and amino terminal proBNP in the emergency diagnosis of heart failure," Heart, vol. 91, no. 5, pp. 606-612, 2005.

[126] A. E. Berezin, "Biomarkers for cardiovascular risk in patients with diabetes," Heart, vol. 102, no. 24, pp. 1939-1941, 2016.

[127] M. S. Kim, T. D. Jeong, S. B. Han, W. K. Min, and J. J. Kim, "Role of soluble ST2 as a prognostic marker in patients with acute heart failure and renal insufficiency," Journal of Korean Medical Science, vol. 30, no. 5, pp. 569-575, 2015.

[128] D. A. Pascual-Figal, J. Ordoñez-Llanos, P. L. Tornel et al., "Soluble ST2 for predicting sudden cardiac death in patients with chronic heart failure and left ventricular systolic dysfunction," Journal of the American College of Cardiology, vol. 54, no. 23, pp. 2174-2179, 2009.

[129] M. Emdin, A. Aimo, G. Vergaro et al., "sST2 Predicts Outcome in Chronic Heart Failure Beyond NT-proBNP and High- Sensitivity Troponin T," Journal of the American College of Cardiology, vol. 72, no. 19, pp. 2309-2320, 2018.

[130] E. O’Meara, M. F. Prescott, B. Claggett et al., "Independent prognostic value of serum soluble ST2 measurements in patients with heart failure and a reduced ejection fraction in 
the PARADIGM-HF trial (Prospective Comparison of ARNI With ACEI to Determine Impact on Global Mortality and Morbidity in Heart Failure)," Circulation: Heart Failure, vol. 11, no. 5, article e004446, 2018.

[131] J. Lassus, E. Gayat, C. Mueller et al., "Incremental value of biomarkers to clinical variables for mortality prediction in acutely decompensated heart failure: the Multinational Observational Cohort on Acute Heart Failure (MOCA) study," International Journal of Cardiology, vol. 168, no. 3, pp. 2186-2194, 2013.

[132] W. P. Huang, X. Zheng, L. He, X. Su, C. W. Liu, and M. X. $\mathrm{Wu}$, "Role of soluble ST2 levels and beta-blockers dosage on cardiovascular events of patients with unselected STsegment elevation myocardial infarction," Chinese Medical Journal, vol. 131, no. 11, pp. 1282-1288, 2018.

[133] Y. Michowitz, Y. Arbel, D. Wexler et al., "Predictive value of high sensitivity CRP in patients with diastolic heart failure," International Journal of Cardiology, vol. 125, no. 3, pp. 347351, 2008.

[134] J. P. Araújo, P. Lourenço, A. Azevedo et al., "Prognostic value of high-sensitivity C-reactive protein in heart failure: a systematic review," Journal of Cardiac Failure, vol. 15, no. 3, pp. 256-266, 2009.

[135] A. P. Kalogeropoulos, W. H. W. Tang, A. Hsu et al., "Highsensitivity C-reactive protein in acute heart failure: insights from the ASCEND-HF trial," Journal of Cardiac Failure, vol. 20, no. 5, pp. 319-326, 2014.

[136] K. Huynh, B. Van Tassell, and S. L. Chow, "Predicting therapeutic response in patients with heart failure: the story of C-reactive protein," Expert Review of Cardiovascular Therapy, vol. 13, no. 2, pp. 153-161, 2015, Epub 2015 Jan 12.

[137] K. C. Wollert, T. Kempf, and L. Wallentin, "Growth differentiation factor 15 as a biomarker in cardiovascular disease," Clinical Chemistry, vol. 63, no. 1, pp. 140-151, 2017.

[138] T. Kempf, M. Eden, J. Strelau et al., "The transforming growth factor-beta superfamily member growthdifferentiation factor- 15 protects the heart from ischemia/reperfusion injury," Circulation Research, vol. 98, no. 3, pp. 351-360, 2006.

[139] A. Rohatgi, P. Patel, S. R. Das et al., "Association of growth differentiation factor-15 with coronary atherosclerosis and mortality in a young, multiethnic population: observations from the Dallas Heart Study," Clinical Chemistry, vol. 58, no. 1, pp. 172-182, 2012.

[140] P. Bettencourt, J. Ferreira-Coimbra, P. Rodrigues et al., "Towards a multi-marker prognostic strategy in acute heart failure: a role for GDF-15,” ESC Heart Failure, vol. 5, no. 6, pp. 1017-1022, 2018.

[141] T. Kempf, S. von Haehling, T. Peter et al., "Prognostic utility of growth differentiation factor-15 in patients with chronic heart failure," Journal of the American College of Cardiology, vol. 50, no. 11, pp. 1054-1060, 2007.

[142] M. M. Y. Chan, R. Santhanakrishnan, J. P. C. Chong et al., "Growth differentiation factor 15 in heart failure with preserved vs. reduced ejection fraction," European Journal of Heart Failure, vol. 18, no. 1, pp. 81-88, 2016.

[143] I. S. Anand, T. Kempf, T. S. Rector et al., "Serial measurement of growth-differentiation factor-15 in heart failure: relation to disease severity and prognosis in the Valsartan Heart Failure Trial," Circulation, vol. 122, no. 14, pp. 1387-1395, 2010.
[144] D. J. Lok, I. J. T. Klip, S. I. Lok et al., "Incremental Prognostic Power of Novel Biomarkers (Growth-Differentiation Factor15, High-Sensitivity C-Reactive Protein, Galectin-3, and High- Sensitivity Troponin-T) in Patients With Advanced Chronic Heart Failure," The American Journal of Cardiology, vol. 112, no. 6, pp. 831-837, 2013.

[145] B. G. Demissei, G. Cotter, M. F. Prescott et al., “A multimarker multi-time point-based risk stratification strategy in acute heart failure: results from the RELAX-AHF trial," European Journal of Heart Failure, vol. 19, no. 8, pp. 1001-1010, 2017.

[146] J. Li, Y. Cui, A. Huang et al., "Additional diagnostic value of growth differentiation factor-15 (GDF-15) to N-terminal Btype natriuretic peptide (NT-proBNP) in patients with different stages of heart failure," Medical Science Monitor, vol. 24, pp. 4992-4999, 2018.

[147] L. B. Daniels and Q. M. Bui, "Should a high Gal-3 have us scared stiff?," Journal of the American College of Cardiology, vol. 73, no. 18, pp. 2296-2298, 2019.

[148] J. Dumic, S. Dabelic, and M. Flogel, "Galectin-3: an openended story," Biochim Biophys Acta, vol. 1760, no. 4, pp. 616-635, 2006.

[149] H. Sano, D. K. Hsu, J. R. Apgar et al., "Critical role of galectin3 in phagocytosis by macrophages," Journal of Clinical Investigation, vol. 112, no. 3, pp. 389-397, 2003.

[150] A. Krzeslak and A. Lipinska, "Galectin-3 as a multifunctional protein," Cellular \& Molecular Biology Letters, vol. 9, no. 2, pp. 305-328, 2004.

[151] U. C. Sharma, S. Pokharel, T. J. van Brakel et al., "Galectin-3 marks activated macrophages in failure-prone hypertrophied hearts and contributes to cardiac dysfunction," Circulation, vol. 110, no. 19, pp. 3121-3128, 2004.

[152] L. Calvier, M. Miana, P. Reboul et al., "Galectin-3 mediates aldosterone-induced vascular fibrosis," Arteriosclerosis, Thrombosis, and Vascular Biology, vol. 33, no. 1, pp. 67-75, 2013.

[153] L. Yu, W. P. T. Ruifrok, M. Meissner et al., "Genetic and pharmacological inhibition of galectin-3 prevents cardiac remodeling by interfering with myocardial fibrogenesis," Circulation: Heart Failure, vol. 6, no. 1, pp. 107-117, 2013.

[154] R. A. de Boer, A. A. Voors, P. Muntendam, W. H. van Gilst, and D. J. van Veldhuisen, "Galectin-3: a novel mediator of heart failure development and progression," European Journal of Heart Failure, vol. 11, no. 9, pp. 811-817, 2009.

[155] K. Karatolios, G. Chatzis, V. Holzendorf et al., "Galectin-3 as a Predictor of Left Ventricular Reverse Remodeling in Recent- Onset Dilated Cardiomyopathy," Disease Markers, vol. 2018, Article ID 2958219, 7 pages, 2018.

[156] R. A. de Boer, L. Yu, and D. J. van Veldhuisen, "Galectin-3 in cardiac remodeling and heart failure," Current Heart Failure Reports, vol. 7, no. 1, pp. 1-8, 2010.

[157] R. A. de Boer, D. J. A. Lok, T. Jaarsma et al., "Predictive value of plasma galectin-3 levels in heart failure with reduced and preserved ejection fraction," Annals of Medicine, vol. 43, no. 1, pp. 60-68, 2010.

[158] D. J. Lok, S. I. Lok, P. W. Bruggink-André de la Porte et al., "Galectin-3 is an independent marker for ventricular remodeling and mortality in patients with chronic heart failure," Clinical Research in Cardiology, vol. 102, no. 2, pp. 103-110, 2013.

[159] L. C. van Vark, I. Lesman-Leegte, S. J. Baart et al., "Prognostic value of serial galectin-3 measurements in patients with acute 
heart failure," Journal of the American Heart Association, vol. 6, no. 12, article e003700, 2017.

[160] A. Bayes-Genis, M. de Antonio, J. Vila et al., "Head-to-head comparison of 2 myocardial fibrosis biomarkers for longterm heart failure risk stratification: ST2 versus galectin-3," Journal of the American College of Cardiology, vol. 63, no. 2, pp. 158-166, 2014, Epub 2013 Sep 24.

[161] A. Chen, W. Hou, Y. Zhang, Y. Chen, and B. He, "Prognostic value of serum galectin-3 in patients with heart failure: A meta- analysis," International Journal of Cardiology, vol. 182, pp. 168-170, 2015.

[162] J. Meluzin, J. Tomandl, H. Podrouzkova et al., "Can markers of collagen turnover or other biomarkers contribute to the diagnostics of heart failure with normal left ventricular ejection fraction?," Biomedical Papers, vol. 157, no. 4, pp. 331339, 2013, Epub 2013 Feb 14.

[163] R. Martos, J. Baugh, M. Ledwidge et al., "Diagnosis of heart failure with preserved ejection fraction: improved accuracy with the use of markers of collagen turnover," European Journal of Heart Failure, vol. 11, no. 2, pp. 191-197, 2009.

[164] F. H. Rutten, M. J. Cramer, and W. J. Paulus, "Heart failure with preserved ejection fraction: diastolic heart failure," Nederlands Tijdschrift voor Geneeskunde, vol. 156, no. 45, p. A5315, 2012.

[165] S. de Denus, J. Lavoie, A. Ducharme et al., "Differences in biomarkers in patients with heart failure with a reduced vs a preserved left ventricular ejection fraction," Canadian Journal of Cardiology, vol. 28, no. 1, pp. 62-68, 2012.

[166] N. Maharaj, B. K. Khandheria, E. Libhaber et al., "Relationship between left ventricular twist and circulating biomarkers of collagen turnover in hypertensive patients with heart failure," Journal of the American Society of Echocardiography, vol. 27, no. 10, pp. 1064-1071, 2014.

[167] T. A. Zelniker, P. Jarolim, B. M. Scirica et al., "Biomarker of collagen turnover (C-terminal telopeptide) and prognosis in patients with non-ST-elevation acute coronary syndromes," Journal of the American Heart Association, vol. 8, no. 9, article e011444, 2019.

[168] K. Nagao, T. Inada, A. Tamura et al., "Circulating markers of collagen types I, III, and IV in patients with dilated cardiomyopathy: relationships with myocardial collagen expression," ESC Heart Failure, vol. 5, no. 6, pp. 1044-1051, 2018.

[169] A. M. Dupuy, N. Kuster, C. Curinier et al., "Exploring collagen remodeling and regulation as prognosis biomarkers in stable heart failure," Clinica Chimica Acta, vol. 490, pp. 167-171, 2019.

[170] M. Volpe, M. Carnovali, and V. Mastromarino, "The natriuretic peptides system in the pathophysiology of heart failure: from molecular basis to treatment," Clinical Science, vol. 130, no. 2, pp. 57-77, 2016.

[171] M. Volpe, S. Rubattu, and J. Burnett, "Natriuretic peptides in cardiovascular diseases: current use and perspectives," European Heart Journal, vol. 35, no. 7, pp. 419-425, 2014.

[172] R. Kerkelä, J. Ulvila, and J. Magga, "Natriuretic peptides in the regulation of cardiovascular physiology and metabolic events," Journal of the American Heart Association, vol. 4, no. 10, article e002423, 2015.

[173] C. Calvieri, S. Rubattu, and M. Volpe, "Molecular mechanisms underlying cardiac antihypertrophic and antifibrotic effects of natriuretic peptides," Journal of Molecular Medicine, vol. 90, no. 1, pp. 5-13, 2012.
[174] D. K. Gupta and T. J. Wang, "Natriuretic peptides and cardiometabolic health," Circulation Journal, vol. 79, no. 8, pp. 1647-1655, 2015.

[175] L. R. Potter, "Natriuretic peptide metabolism, clearance and degradation,” FEBS Journal, vol. 278, no. 11, pp. 1808-1817, 2011.

[176] R. D’Alessandro, D. Masarone, A. Buono et al., "Natriuretic peptides: molecular biology, pathophysiology and clinical implications for the cardiologist," Future Cardiology, vol. 9, no. 4, pp. 519-534, 2013.

[177] Z. Cao, Y. Jia, and B. Zhu, "BNP and NT-proBNP as diagnostic biomarkers for cardiac dysfunction in both clinical and forensic medicine," International Journal of Molecular Sciences, vol. 20, no. 8, p. 1820, 2019.

[178] M. A. Alpert, C. J. Lavie, H. Agrawal, K. B. Aggarwal, and S. A. Kumar, "Obesity and heart failure: epidemiology, pathophysiology, clinical manifestations, and management," Translational Research, vol. 164, no. 4, pp. 345356, 2014.

[179] E. H. Nah, S. Y. Kim, S. Cho, S. Kim, and H. I. Cho, "Plasma NT-proBNP levels associated with cardiac structural abnormalities in asymptomatic health examinees with preserved ejection fraction: a retrospective cross-sectional study," BMJ Open, vol. 9, no. 4, article e026030, 2019.

[180] U. L. Faxén, L. H. Lund, N. Orsini et al., "N-terminal pro-Btype natriuretic peptide in chronic heart failure: the impact of sex across the ejection fraction spectrum," International Journal of Cardiology, vol. 287, pp. 66-72, 2019, Epub 2019 Apr 11.

[181] F. S. Gaborit, C. Kistorp, T. Kümler et al., "Prevalence of early stages of heart failure in an elderly risk population: the Copenhagen Heart Failure Risk Study," Open Heart, vol. 6, no. 1, article e000840, 2019.

[182] Z. J. Han, X. D. Wu, J. J. Cheng et al., "Diagnostic accuracy of natriuretic peptides for heart failure in patients with pleural effusion: a systematic review and updated meta-analysis," PLoS One, vol. 10, no. 8, article e0134376, 2015.

[183] H.-P. B.-L. Rocca and S. S.-v. Wijk, "Natriuretic peptides in chronic heart failure," Cardiac Failure Review, vol. 5, no. 1, pp. 44-49, 2019.

[184] P. L. Myhre, M. Vaduganathan, B. Claggett et al., "B-type natriuretic peptide during treatment with sacubitril/valsartan: the PARADIGM-HF Trial," Journal of the American College of Cardiology, vol. 73, no. 11, pp. 1264-1272, 2019.

[185] S. Stienen, K. Salah, A. H. Moons et al., "NT-proBNP (N-terminal pro-B-type natriuretic peptide)-guided therapy in acute decompensated heart failure: PRIMA II randomized controlled trial (can NT-proBNP-guided therapy during hospital admission for acute decompensated heart failure reduce mortality and readmissions?)," Circulation, vol. 137, no. 16, pp. 1671-1683, 2018, Epub 2017 Dec 14.

[186] G. M. Felker, K. J. Anstrom, K. F. Adams et al., "Effect of natriuretic peptide-guided therapy on hospitalization or cardiovascular mortality in high-risk patients with heart failure and reduced ejection fraction: a randomized clinical trial," JAMA, vol. 318, no. 8, pp. 713-720, 2017.

[187] N. G. Morgenthaler, J. Struck, C. Alonso, and A. Bergmann, "Assay for the measurement of copeptin, a stable peptide derived from the precursor of vasopressin," Clinical Chemistry, vol. 52, no. 1, pp. 112-119, 2006. 
[188] L. Dobša and K. C. Edozien, "Copeptin and its potential role in diagnosis and prognosis of various diseases," Biochemia Medica, vol. 23, no. 2, pp. 172-192, 2013.

[189] D. Bolignano, A. Cabassi, E. Fiaccadori et al., "Copeptin (CTproAVP), a new tool for understanding the role of vasopressin in pathophysiology," Clinical Chemistry and Laboratory Medicine (CCLM), vol. 52, no. 10, pp. 1447-1456, 2014.

[190] N. G. Morgenthaler, "Copeptin: a biomarker of cardiovascular and renal function," Congestive Heart Failure, vol. 16, Suppl 1, pp. S37-S44, 2010.

[191] W. J. Tu, G. Z. Ma, Y. Ni et al., “Copeptin and NT-proBNP for prediction of all-cause and cardiovascular death in ischemic stroke," Neurology, vol. 88, no. 20, pp. 1899-1905, 2017.

[192] W. Fenske, C. Wanner, B. Allolio et al., "Copeptin levels associate with cardiovascular events in patients with ESRD and type 2 diabetes mellitus," Journal of the American Society of Nephrology, vol. 22, no. 4, pp. 782-790, 2011.

[193] I. Tasevska, S. Enhorning, M. Persson, P. M. Nilsson, and O. Melander, "Copeptin predicts coronary artery disease cardiovascular and total mortality," Heart, vol. 102, no. 2, pp. 127-132, 2016.

[194] S. S. Bhandari, I. Loke, J. E. Davies, I. B. Squire, J. Struck, and L. L. Ng, "Gender and renal function influence plasma levels of copeptin in healthy individuals," Clinical Science, vol. 116, no. 3, pp. 257-263, 2009.

[195] G. Velho, S. Ragot, R. el Boustany et al., "Plasma copeptin, kidney disease, and risk for cardiovascular morbidity and mortality in two cohorts of type 2 diabetes," Cardiovascular Diabetology, vol. 17, no. 1, p. 110, 2018.

[196] A. E. Berezin, "Up-to-date clinical approaches of biomarkers' use in heart failure," Biomedical Research and Therapy, vol. 4, no. 6, pp. 1344-1370, 2017.

[197] K. Kitamura, K. Kangawa, and T. Eto, "Adrenomedullin and PAMP: discovery, structures, and cardiovascular functions," Microscopy Research and Technique, vol. 57, no. 1, pp. 3-13, 2002.

[198] T. Nishikimi and Y. Nakagawa, "Adrenomedullin as a biomarker of heart failure," Heart Failure Clinics, vol. 14, no. 1, pp. 49-55, 2018, Epub 2017 Oct 7.

[199] K. Kobayashi, K. Kitamura, N. Hirayama et al., "Increased plasma adrenomedullin in acute myocardial infarction," American Heart Journal, vol. 131, no. 4, pp. 676-680, 1996.

[200] Y. Miyao, T. Nishikimi, Y. Goto et al., "Increased plasma adrenomedullin levels in patients with acute myocardial infarction in proportion to the clinical severity," Heart, vol. 79, no. 1, pp. 39-44, 1998.

[201] O. S. Dhillon, S. Q. Khan, H. K. Narayan et al., "Prognostic Value of Mid-Regional Pro-Adrenomedullin Levels Taken on Admission and Discharge in Non-ST-Elevation Myocardial Infarction: The LAMP (Leicester Acute Myocardial Infarction Peptide) II Study," Journal of the American College of Cardiology, vol. 56, no. 2, pp. 125-133, 2010.

[202] I. T. Klip, A. A. Voors, S. D. Anker et al., "Prognostic value of mid-regional pro-adrenomedullin in patients with heart failure after an acute myocardial infarction," Heart, vol. 97, no. 11, pp. 892-898, 2011.

[203] S. Q. Khan, R. J. O’Brien, J. Struck et al., "Prognostic value of midregional pro-adrenomedullin in patients with acute myocardial infarction: the LAMP (Leicester Acute Myocardial Infarction Peptide) study," Journal of the American College of Cardiology, vol. 49, no. 14, pp. 1525-1532, 2007.
[204] A. Maisel, C. Mueller, R. Nowak et al., "Mid-region prohormone markers for diagnosis and prognosis in acute dyspnea: results from the BACH (Biomarkers in Acute Heart Failure) trial," Journal of the American College of Cardiology, vol. 55, no. 19, pp. 2062-2076, 2010.

[205] A. C. Falkentoft, R. Rørth, K. Iversen et al., "MR-proADM as a prognostic marker in patients with ST-segment-elevation myocardial infarction-DANAMI-3 (a Danish Study of Optimal Acute Treatment of Patients With STEMI) Substudy," Journal of the American Heart Association, vol. 7, no. 11, article e008123, 2018.

[206] A. Gegenhuber, J. Struck, B. Dieplinger et al., "Comparative evaluation of B-type natriuretic peptide, mid-regional proA-type natriuretic peptide, mid-regional pro-adrenomedullin, and copeptin to predict 1-year mortality in patients with acute destabilized heart failure," Journal of Cardiac Failure, vol. 13, no. 1, pp. 42-49, 2007.

[207] O. Melander, C. Newton-Cheh, P. Almgren et al., "Novel and conventional biomarkers for prediction of incident cardiovascular events in the community," JAMA, vol. 302, no. 1, pp. 49-57, 2009.

[208] J. T. Neumann, S. Tzikas, A. Funke-Kaiser et al., “Association of MR-proadrenomedullin with cardiovascular risk factors and subclinical cardiovascular disease," Atherosclerosis, vol. 228, no. 2, pp. 451-459, 2013.

[209] K. S. Shah, N. A. Marston, C. Mueller et al., "Midregional proadrenomedullin predicts mortality and major adverse cardiac events in patients presenting with chest pain: results from the CHOPIN trial," Academic Emergency Medicine, vol. 22, no. 5, pp. 554-563, 2015.

[210] C. Sinning, F. Ojeda, P. S. Wild et al., "Midregional proadrenomedullin and growth differentiation factor-15 are not influenced by obesity in heart failure patients," Clinical Research in Cardiology, vol. 106, no. 6, pp. 401-410, 2017.

[211] S. Dangwal, K. Schimmel, A. Foinquinos, K. Xiao, and T. Thum, "Noncoding RNAs in heart failure," in Heart Failure, vol. 243 of Handbook of Experimental Pharmacology, pp. 423-445, Springer, Cham, 2017.

[212] E. L. Vegter, P. van der Meer, L. J. de Windt, Y. M. Pinto, and A. A. Voors, "MicroRNAs in heart failure: from biomarker to target for therapy," European Journal of Heart Failure, vol. 18, no. 5, pp. 457-468, 2016, Epub 2016 Feb 11.

[213] W. Li, M. Liu, C. Zhao et al., "MiR-1/133 attenuates cardiomyocyte apoptosis and electrical remodeling in mice with viral myocarditis," Cardiology Journal, 2013, [Epub ahead of print].

[214] A. Jodati, S. M. Pirouzpanah, N. Fathi Maroufi et al., "Different expression of Micro RNA-126, 133a and 145 in aorta and saphenous vein samples of patients undergoing coronary artery bypass graft surgery," Journal of Cardiovascular and Thoracic Research, vol. 11, no. 1, pp. 43-47, 2019.

[215] Y. Xiao, J. Zhao, J. P. Tuazon, C. V. Borlongan, and G. Yu, "MicroRNA-133a and myocardial infarction," Cell Transplantation, vol. 28, no. 7, pp. 831-838, 2019, [Epub ahead of print].

[216] J. Song, Q. Xie, L. Wang et al., “The TIR/BB-loop mimetic AS-1 prevents Ang II-induced hypertensive cardiac hypertrophy via NF- $\kappa$ B dependent downregulation of miRNA-143," Scientific Reports, vol. 9, no. 1, p. 6354, 2019.

[217] P. Shah, M. R. Bristow, and J. D. Port, "MicroRNAs in heart failure cardiac transplantation, and myocardial recovery: 
biomarkers with therapeutic potential," Current Heart Failure Reports, vol. 14, no. 6, pp. 454-464, 2017.

[218] R. Verjans, W. J. A. Derks, K. Korn et al., "Functional screening identifies microRNAs as multi-cellular regulators of heart failure," Scientific Reports, vol. 9, no. 1, p. 6055, 2019.

[219] A. Samman Tahhan, M. Hammadah, M. Raad et al., "Progenitor cells and clinical outcomes in patients with acute coronary syndromes," Circulation Research, vol. 122, no. 11, pp. 1565-1575, 2018.

[220] E. Shantsila, B. J. Wrigley, A. Shantsila, L. D. Tapp, P. S. Gill, and G. Y. H. Lip, "Monocyte-derived and CD34+/KDR+ endothelial progenitor cells in heart failure," Journal of Thrombosis and Haemostasis, vol. 10, no. 7, pp. 1252-1261, 2012.

[221] E. Nollet, V. Y. Hoymans, I. R. Rodrigus et al., "Bone marrow-derived progenitor cells are functionally impaired in ischemic heart disease," Journal of Cardiovascular Translational Research, vol. 9, no. 4, pp. 266-278, 2016.

[222] A. E. Berezin and A. A. Kremzer, "Circulating endothelial progenitor cells as markers for severity of ischemic chronic heart failure," Journal of Cardiac Failure, vol. 20, no. 6, pp. 438-447, 2014.

[223] A. E. Berezin, A. A. Kremzer, Y. V. Martovitskaya et al., "The utility of biomarker risk prediction score in patients with chronic heart failure," International Journal of Clinical and Experimental Medicine, vol. 8, no. 10, pp. 18255-18264, 2015.

[224] A. E. Berezin, A. A. Kremzer, T. A. Samura et al., "Predictive value of apoptotic microparticles to mononuclear progenitor cells ratio in advanced chronic heart failure patients," Journal of Cardiology, vol. 65, no. 5, pp. 403-411, 2015.

[225] J. A. Suárez-Cuenca, R. Robledo-Nolasco, M. A. AlcántaraMeléndez et al., "Coronary circulating mononuclear progenitor cells and soluble biomarkers in the cardiovascular prognosis after coronary angioplasty," Journal of Cellular and Molecular Medicine, vol. 23, no. 7, 2019. 